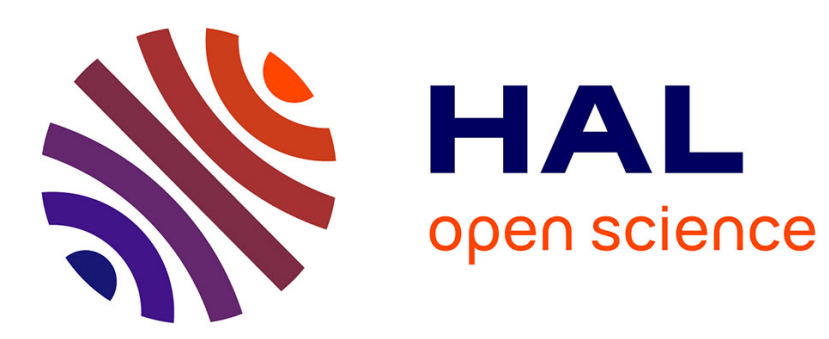

\title{
Simulation of energy transfers in waves generated by granular slides
}

Lucie Clous, Stéphane Abadie

\section{To cite this version:}

Lucie Clous, Stéphane Abadie. Simulation of energy transfers in waves generated by granular slides. Landslides, 2019, 16 (9), pp.1663-1679. 10.1007/s10346-019-01180-0 . hal-02157085

\section{HAL Id: hal-02157085 \\ https://hal-univ-pau.archives-ouvertes.fr/hal-02157085}

Submitted on 15 Jun 2019

HAL is a multi-disciplinary open access archive for the deposit and dissemination of scientific research documents, whether they are published or not. The documents may come from teaching and research institutions in France or abroad, or from public or private research centers.
L'archive ouverte pluridisciplinaire HAL, est destinée au dépôt et à la diffusion de documents scientifiques de niveau recherche, publiés ou non, émanant des établissements d'enseignement et de recherche français ou étrangers, des laboratoires publics ou privés. 


\title{
Simulation of energy transfers in waves generated by granular slides
}

\author{
Lucie Clous and Stéphane Abadie \\ Université de Pau et des Pays de l'Adour, E2S UPPA, Laboratoire des Sciences de \\ l'Ingénieur appliquées à la Mécanique et au génie Electrique, EA4581, 64600, ANGLET, \\ FRANCE
}

June 15, 2019

\begin{abstract}
This paper presents a multi-fluid Navier-Stokes modelling of the waves generated by two granular slides (subaerial and submarine) which were previously studied experimentally and a pure synthetic submarine case used for results interpretation. In the numerical model, air and water are considered as Newtonian fluids. The slide is modelled as a Newtonian fluid whose viscosity is adjusted to fit the experimental results. Once the viscosity is adjusted, the first and the second waves are shown to be accurately reproduced by the model even though the computed slide is slower. For the subaerial case, the viscosity value found is shown to be consistent with the granular $\mu(I)$ rheological law. The second part of this work focuses on the energy transfers between a slide and its generated waves. Energy balance is computed in each phase. The wave energy is evaluated in the wave propagation zone. Energy dissipation, kinematic and potential energies are taken into account in the computation of energy transfer ratio allowing for a better understanding of the phenomena. In light of these results, the wave train generation process is discussed as well as the importance of the slide dynamics in the wave generation stage. The amount of energy transferred to wave is not constant with time and the transfer rate depends strongly on the definition of this rate as well as the case considered. For instance, in the subaerial case simulated, the energy transferred to surface waves is $30 \%$ of the energy transferred to water at the time the transfer stops, but this conversion rate is only equal to $4 \%$ of the overall available potential slide energy at the end of the process. For the two submarine cases simulated, the corresponding values, equal in both cases, are $2 \%$ and $1 \%$, respectively. The simulation results also show that the slide energy is transferred to the water in a short period of time at the beginning whatever the case considered. This observation may be related to the initial nil slide velocity (subaerial case) and the relatively large slope values considered (both cases). Nevertheless, the results illustrate the importance of accurate simulation of the slide dynamics within the wave generation process.
\end{abstract}

Keywords landslide-generated waves, numerical simulations, multiphase model, energy transfer, subaerial slide, submarine slide, granular rheology.

\section{Introduction}

The generation of waves by landslides, whether subaerial or submarine, is a complex phenomenon which has been studied extensively in the last twenty years. For subaerial slides, the free surface is very perturbed, sometimes involving vortex creation, wave breaking, and air entrainment (Fritz et al., 2004). Submarine slides involve less interactions with the water free surface. Nevertheless, the coupling between the slide and water cannot always be neglected, especially if the slide is shallow and has a relative density close to one (Jiang and LeBlond, 1992).

Identifying the main slide parameters contributing to the wave generation has been the focus of several former studies including Fritz et al. (2004), Ataie-Ashtiani and Nik-Khah (2008), Heller and Hager (2010) and Mulligan and Take (2017). These studies found the slide mass, thickness and impact velocity as being 
particularly important parameters for subaerial slide cases. For submarine slides, the slide submergence depth appears critical. Recently, Lo and Liu (2017) found that the slide area, in two-dimensions (2D), is the relevant parameter explaining the wave height generated, whatever the slide shape.

Experimental studies have been carried out with rigid blocks (Heinrich, 1992; Liu et al., 2005; AtaieAshtiani and Najafi-Jilani, 2008; Ataie-Ashtiani and Nik-Khah, 2008) or granular media (Fritz et al., 2004; Viroulet et al., 2014). The importance of the slide deformation has been highlighted by AtaieAshtiani and Najafi-Jilani (2008) and Ataie-Ashtiani and Nik-Khah (2008). They show that with similar initial rigid and deformable slides, the maximum wave crest amplitude is reduced from $15 \%$ to $25 \%$ for submarine cases and from $25 \%$ up to $35 \%$ for subaerial cases in the deformable slide case taking the rigid slide case as reference.

Numerical models are often employed to complement experimental studies to overcome some experimental shortcomings such as the lack of information on the velocity fields in the slide and in water or scale effects and build applicable predictive tools. These models may rely on different types of equations including shallow water models (Jiang and LeBlond, 1992), Boussinesq-type equations (Lynett and Liu, 2002; Watts et al., 2003), and Navier-Stokes equations (Abadie et al., 2010) which are specially required in the case of subaerial slides with a more complex nature.

The slide model can be described as a solid block (Abadie et al., 2010), a non-viscous fluid (AtaieAshtiani and Nik-Khah, 2008; Abadie et al., 2012), a viscous fluid (Jiang and LeBlond, 1992), or a viscoplastic fluid with different rheologies such as Bingham model (Jiang and LeBlond, 1993; Viroulet, 2013). A granular flow can also be modelled as a Coulomb-like continuum (Mangeney-Castelnau et al., 2003; Kelfoun et al., 2010; Ma et al., 2015; Yavari-Ramshe and Ataie-Ashtiani, 2017), or with a DEM (Discrete Element Method) code which computes the slide displacement coupled to a CFD (Computational Fluid Dynamics) model (Zhao et al., 2016). More simply, the Newtonian fluid model considers the slide as a fluid with a constant viscosity (Grilli et al., 2017). This constant viscosity can be seen as a first approximation of a granular law like the $\mu(I)$-rheology (GDR MiDi, 2004) for instance. The latter is a more complex model with a threshold and an equivalent viscosity varying in space and time. It is used for the modelling of dense dry granular flow and has been generalized by Cassar et al. (2005) for non-dry granular flow.

A few studies have been focused on the energy transfer between a slide and its generated waves. Depending on the study, the considered landslide energy can be the initial potential energy, generally for submarine slides (Jiang and LeBlond, 1992; Ruff, 2003) or the kinetic energy at the impact, for subaerial slides (Watts, 1997; Fritz et al., 2004). For the computation of the wave energy, some authors computed only the potential energy and considered that there is an equipartition with the kinetic energy (Fritz et al., 2004; Ataie-Ashtiani and Nik-Khah, 2008). However, Fritz et al. (2004) pointed out that this hypothesis is not correct near the impact zone of subaerial slides. Moreover, it is common for landslides to generate highly non-linear waves and in this case, to have a kinetic energy larger than the potential energy (Heller and Hager, 2011; Heller et al., 2016). For this reason, both potential and kinetic wave energies were computed by Heller et al. (2016) and also on a part of the domain by Sue et al. (2011). In 2D channel, the conversion of energy from the slide to the waves is evaluated to be $4-50 \%$ for a subaerial slide (Fritz et al., 2004) and between $2 \%$ and $14 \%$ for a submarine slide (Jiang and LeBlond, 1992; Watts, 1997; Sue et al., 2011). Similar conversion ratios have been identified in experiments involving radial wave propagation due to larger flume to slide widths ratio (Ataie-Ashtiani and Nik-Khah, 2008; Ataie-Ashtiani and Najafi-Jilani, 2008; Yavari-Ramshe and Ataie-Ashtiani, 2019), with a range from $1 \%$ to $40 \%$ for subaerial slides and around $4 \%$ for submarine slides. If most of these results were obtained experimentally, recent attempts to compute energy transfers in advanced numerical models have also to be aknowledged. For instance, Ma et al. (2015) proposed a new two-layer model for granular landslide motion and tsunami wave generation and studied the time evolution of the tsunami potential and kinetic energy.

In the present paper, the wave generation process is simulated for three cases of granular slides (subaerial and submarine) using the incompressible Navier-Stokes Volume Of Fluid (VOF) model THETIS (Abadie et al., 2010).

The granular slide is modelled as a fluid based on a simple viscous Newtonian approximation. This simple law, when properly adjusted, can be seen as a very coarse approximation of the more elaborated non-Newtonian $\mu(I)$-rheology with which the model is also compared. A comparison with the existing physical measurements allows to calibrate the model. Then, the potential and the kinetic energies and the energy dissipation are computed at each time step in the water and the slide, giving the whole process 
of energy transfers from the slide to the waves. Finally, the wave generation process is discussed.

\section{Numerical model}

THETIS is a multifluid Navier-Stokes (NS) solver developed by the TREFLE department of the I2M Laboratory at Bordeaux, France. The present numerical simulations are based on the direct solution of the incompressible NS equations for water, air, and the slide, with a two-step incremental projection method (Goda, 1979). THETIS can be considered as a one-fluid model as only one velocity is defined at each point of the mesh and there is no mixing between the three considered fluid of water, air, and slide. An equivalent fluid is defined at each mesh cell and its characteristics (density and viscosity) vary in space. In each simulation stage, the time step is updated based on the considered limit for the Courant number (Courant et al., 1967) ( $\frac{u \Delta t}{\Delta x}$ with $u$ the magnitude of the velocity, $\Delta t$ the time step, $\Delta x$ the length interval) to a certain value (generally 0.5$)$.

In addition to the NS equations, two advection equations are solved for the water and the slide particles. The interface tracking is performed by a VOF-TVD method based on the Lax-Wendroff scheme. A more accurate VOF-PLIC method is also available in THETIS but this method is more sensitive and may fail when violent impacts such as in the subaerial case are occurring. The portion of each phase in a cell is defined by a colour function whose value is between 0 and 1. A free slip condition is imposed on the velocities of the left, right and top faces of the domain while a wall condition is imposed on the bottom boundary. The slope is obtained by positioning a porous body (Desombre et al., 2012) with a nil porosity. The computational mesh follows the geometry of each solid part of the domain with a stair-like slope. According to the performed simulations, the mesh resolution $(\Delta x=5 \mathrm{~mm}, \Delta y=2 \mathrm{~mm}$ and $\Delta x=2.85 \mathrm{~mm}, \Delta y=1.25 \mathrm{~mm}$ in the subaerial and submarine case respectively) used is sufficient to limit the effects of the slope irregularities on water and slide flows.

The water, the air, and the slide are firstly modelled as Newtonian fluids, giving one parameter, namely viscosity, for the slide to be calibrated. The granular $\mu(I)$-rheology (GDR MiDi, 2004) has also been implemented in THETIS to model dry dense granular flows. It has been validated by comparing with a dry granular column collapse (Lagrée et al., 2011). The three material-dependant parameters are $I_{0}, \mu_{s}$ and $\Delta \mu$. They define the friction coefficient $\mu(I)$ which only depends on the inertial number $I$. In THETIS, these variables are evaluated on each point of the slide and the viscosity $\eta$ is computed and imposed as the local fluid viscosity value in the NS equations. This gives a viscosity in the slide that is space and time-dependent.

$$
\begin{gathered}
\eta=\max \left(\frac{\mu(I)}{\sqrt{2} D_{2}} p, 0\right) \\
\mu(I)=\mu_{s}+\frac{\Delta \mu}{I_{0} / I+1} \\
I=\frac{d \sqrt{2} D_{2}}{\sqrt{|p| / \rho}}
\end{gathered}
$$

with $D_{2}$ as the second invariant of the strain rate tensor, $p$ pressure, $\mu_{s}, \Delta \mu$, and $I_{0}$ three materialdependent coefficients, $d$ the slide grain diameter and $\rho$ grain density.

So far, it is not possible to use this rheology in conjunction with water. Indeed, even though Cassar et al. (2005) provided a formulation able to predict the behaviour of granular slide in water, it is not easily adaptable to a one-fluid formulation as used in THETIS. In this paper, it will be used as a reference for the first instants of the subaerial case.

\section{$2.1 \quad$ Energy transfers}

The slide potential energy is maximum at the initial time. During the landslide motion, a part of the potential energy is converted into kinetic energy, lost due to the viscous dissipation, and converted into the water kinetic and potential energies including wave energy which is of interest here.

During the simulations, the different energy components are computed in the slide and water in order to better understand the energy transfer process. One of the main objective is to determine the time duration of energy transfer from the slide to waves and the ratio or efficiency of this energy transfer. 
The local formulation of the kinetic energy theorem (Chassaing, 2000) gives (with gravity as the only volume force):

$$
\frac{\partial}{\partial t}\left(\frac{1}{2} \rho V^{2}\right)+\nabla \cdot\left(\frac{1}{2} \rho V^{2} \underline{V}\right)=\rho \underline{V} \cdot \underline{g}+\nabla \cdot \underline{\underline{\tau}} \cdot \underline{V}-\nabla \cdot P \underline{V}+P \nabla \cdot \underline{V}-\underline{\underline{\tau}}: \underline{\underline{D}}
$$

With $\underline{V}$ the local fluid velocity, $P$ the local fluid pressure, $\underline{\tau}$ the shear stress tensor and $\underline{D}$ the strain rate tensor.

By integrating this equation over the fluid volume (i.e., either the slide, water, or air volume), it leads to:

$$
\begin{aligned}
\iiint_{\Omega} \frac{\partial}{\partial t} & \left(\frac{1}{2} \rho V^{2}\right) d \Omega+\iint_{\partial \Omega} \frac{1}{2} \rho V^{2} \underline{V} \cdot \underline{n} d S= \\
& \iiint_{\Omega} \rho \underline{V} \cdot \underline{g} d \Omega+\iint_{\partial \Omega}(\underline{\underline{\tau}} \cdot \underline{V}) \cdot \underline{n} d S-\iint_{\partial \Omega} P \underline{V} \cdot \underline{n} d S+\iiint_{\Omega} P \nabla \cdot \underline{V} d \Omega-\iiint_{\Omega} \underline{\underline{\tau}}: \underline{\underline{D}} d \Omega
\end{aligned}
$$

Using the transport and the continuity equations, it can be written as:

$$
\overbrace{\frac{d}{d t} \iiint_{\Omega}\left(\frac{1}{2} \rho V^{2}\right) d \Omega}^{\mathrm{a}}+\overbrace{\frac{d}{d t} \iiint_{\Omega}(\rho g z) d \Omega}^{\mathrm{b}}+\overbrace{\iint_{\partial \Omega}\left(\frac{1}{2} \rho V^{2}+\rho g z\right)\left(\underline{V}-\underline{V}_{a}\right) \cdot \underline{n} d S}^{\mathrm{c}}+\overbrace{\iint_{\partial \Omega}^{P \underline{V} \cdot \underline{n} d S}}^{\mathrm{d}}=
$$

including kinetic energy variation (a), potential energy variation (b), kinetic and potential energy fluxes through the envelope (c), power of the pressure force on the envelope (d), power of the viscous stress on the envelope (e), power of the pressure force in the volume (f) and rate of the viscous dissipation (g).

(f) vanishes with assuming incompressible fluids. In our case, the envelope velocity is equal to the fluid velocity, therefore (c) is also nil. In the following equation, the left-hand side contains the terms that are calculated while the terms of the right-hand side are deduced from the numerical computation.

$$
\frac{d}{d t}\left(E_{k}+E_{p}\right)+\Phi=\iint_{\partial \Omega} \underline{\underline{\tau}} \cdot \underline{V} d S-\iint_{\partial \Omega} P \underline{V} \cdot \underline{n} d S
$$

Where $E_{k}=\iiint_{\Omega}\left(\frac{1}{2} \rho V^{2}\right) d \Omega, E_{p}=\iiint_{\Omega}(\rho g z) d \Omega, \Phi=\iiint_{\Omega} \underline{\underline{\tau}}: \underline{\underline{D}} d \Omega$.

Equation (7) shows that the changes in the mechanical energy within the fluid volume is due to the total power of the pressure force and shear stress on the fluid envelope. Hence, for water, changes in the total mechanical energy is through the pressure and shear forces induced by the slide along the slide/water interface and vice versa. The balance equation (7) can be written for all three considered fluids, namely the slide, water and air volumes. By computing the air energy, it is remarked that its value does not change significantly during the simulation (section 3.2.1). Therefore, only the variation of energy in the slide and the water portions are considered. Based on the Newton's third law, the right hand sides of the energy balanced equations written for the slide and the water are equal (action-reaction) in each control volume. For this reason, the integrated left-hand terms of this equation computed for one phase represents the energy transferred to the other phase.

$$
\left[\frac{d}{d t}\left(E_{k}+E_{p}\right)+\Phi\right]_{\text {slide }}=-\left[\frac{d}{d t}\left(E_{k}+E_{p}\right)+\Phi\right]_{\text {water }}
$$

The dissipation term $\underline{\tau}: \underline{D}$ is numerically computed for an incompressible Newtonian fluid, namely:

$$
\underline{\underline{\tau}}: \underline{\underline{D}}=2 \eta D_{i j} D_{i j}
$$

By integrating equation ( 7 ) between times 0 and $t$, the transferred energy can be expressed as:

$$
E_{t}(t)=E_{k}(t)+E_{p}(t)-E_{k}(0)-E_{p}(0)+\int_{0}^{t} \Phi d t^{\prime}
$$


where $E_{p}(0)$ is calculated with a vertical reference taken at the bottom.

The total mechanical energy can be defined as $E_{m}=E_{k}+E_{p}$, therefore:

$$
E_{t}(t)=E_{m}(t)-E_{m}(0)+\int_{0}^{t} \Phi d t^{\prime}
$$

This transferred energy is positive if the fluid studied (i.e., slide or water) gains energy, and negative in case of a loss of energy.

Following the previous set of equation, the computation of the different energy components is carried out for the slide, water and air domains separately as follows:

$$
\begin{gathered}
E_{k}=\sum_{i=1}^{N_{X}} \sum_{j=1}^{N_{Y}} \phi(i, j) \frac{1}{2} \rho V^{2}(i, j) \Delta x(i, j) \Delta y(i, j) \\
E_{p}=\sum_{i=1}^{N_{X}} \sum_{j=1}^{N_{Y}} \phi(i, j) \rho g y(i, j) \Delta x(i, j) \Delta y(i, j) \\
\Phi=\sum_{i=1}^{N_{X}} \sum_{j=1}^{N_{Y}} \phi(i, j) 2 \eta D_{k l}(i, j) D_{k l}(i, j) \Delta x(i, j) \Delta y(i, j)
\end{gathered}
$$

where $N_{X}$ and $N_{Y}$ are the number of nodes in the direction $X$ and $Y$ respectively, $\phi$ is the color function value of the phase and $\Delta x$ and $\Delta y$ are the cell size in the direction of $X$ and $Y$, respectively.

To separate the wave energy from the water energy, the water domain is divided into two zones (Figure 1 ) : the generation zone (zone 1) and the propagation zone (zone 2). In the two experimental cases studied, waves propagate faster than the slide (subcritical cases), which helps defining the propagation zone as the one limited by the slide front. Additionally, one may expect zone 1 to be rotational and zone 2 irrotational. The squared vorticity is locally computed to verify this assumption. For this reason, it is considered that the water energy computed in the propagation zone is the wave energy. Kinetic and potential wave energy are computed as follows:

$$
\begin{gathered}
E_{k, w}=\sum_{i=X_{P}}^{N_{X}} \sum_{j=1}^{N_{Y}} \phi(i, j) \frac{1}{2} \rho V(i, j)^{2} \Delta x(i, j) \Delta y(i, j) \\
E_{p, w}=\sum_{i=X_{P}}^{N_{X}} \sum_{j=1}^{N_{Y}} \phi(i, j) \rho g y(i, j) \Delta x(i, j) \Delta y(i, j)-\sum_{i=X_{P}}^{N_{X}} \sum_{j=1}^{Y_{W}} \rho g y(i, j) \Delta x(i, j) \Delta y(i, j)
\end{gathered}
$$

where $X_{P}$ is the abscissa limiting the propagation zone and $Y_{W}$ the initial free surface elevation ordinate.

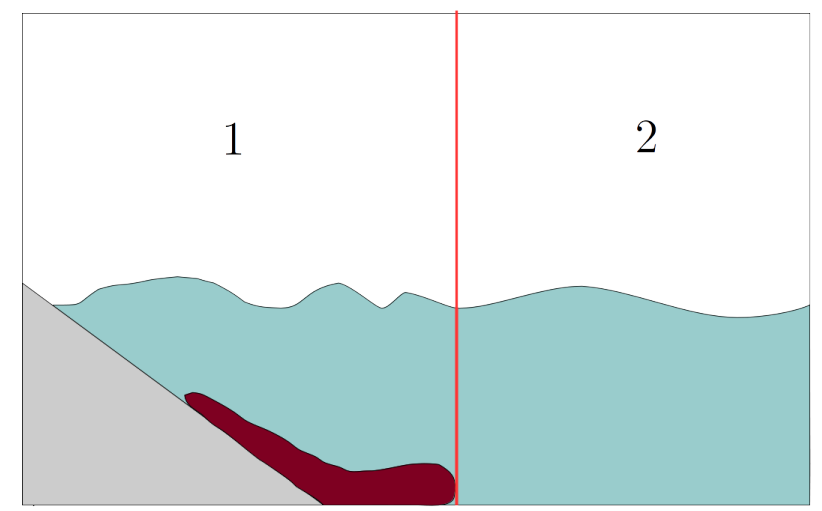

Figure 1: Sketch illustrating assumed generation (1) and propagation (2) zones.

Jiang and LeBlond (1992) characterised the energy transfer ratio as the mechanical energy of the waves divided by the potential energy of the slide. 


$$
\lambda_{J L B}=\frac{E_{m, w}(t)}{E_{p, \text { slide }}(t)-E_{p, \text { slide }}(0)}
$$

However, in the present study with Navier-Stokes simulations, the kinetic energy and the viscous dissipation of the slide can also be easily computed, and therefore the ratio of wave energy on the actual energy transferred to water can be accurately defined.

The instantaneous part of energy transferred to the waves that propagate offshore can then be expressed as:

$$
\lambda(t)=\frac{E_{m, w}(t)}{E_{t, s l i d e}(t)}
$$

with $E_{t, \text { slide }}(t)$ the energy transferred by the slide defined by equation (11).

\section{$3 \quad$ Numerical simulations}

\subsection{Model comparison with experimental results}

\subsubsection{Benchmark test presentation}

The numerical model was tested using two series of available experimental data involving the wave generation by granular slides. The first one (Viroulet et al., 2013), is a subaerial case, initially retained by a gate just at the level of the water free surface with no initial velocity when it impacts the water. It is therefore very different from the experiments of impulse waves generated by granular slides presented by Fritz et al. (2004) and Heller and Hager (2011) where the slide has an impact velocity of $2.06-8.77 \mathrm{~m} / \mathrm{s}$ (Heller and Hager, 2010). The second one (Grilli et al., 2017) is a submarine landslide case.

Case 1 : Subaerial case Viroulet et al. (2013) conducted an experiment in a tank of dimension $220 \mathrm{~cm}$ in length, $40 \mathrm{~cm}$ in height and $20 \mathrm{~cm}$ in width with a water depth of $14.8 \mathrm{~cm}$ (Figure 2(a)). A mass of $2 \mathrm{~kg}$ of spherical glass beads of $1.5 \mathrm{~mm}$ of diameter and density of $2500 \mathrm{~kg} \cdot \mathrm{m}^{-3}$ was placed on a slope of $45^{\circ}$ at the limit with the water free surface. Four gauges of water elevation were disposed at $0.45 \mathrm{~m}$, $0.75 \mathrm{~m}, 1.05 \mathrm{~m}$ and $1.35 \mathrm{~m}$ from the gate.

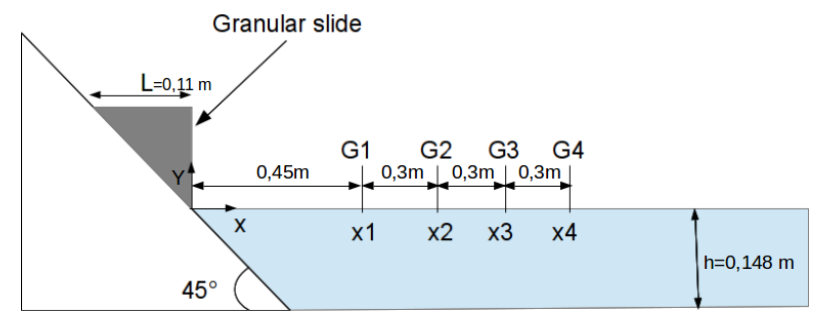

(a)
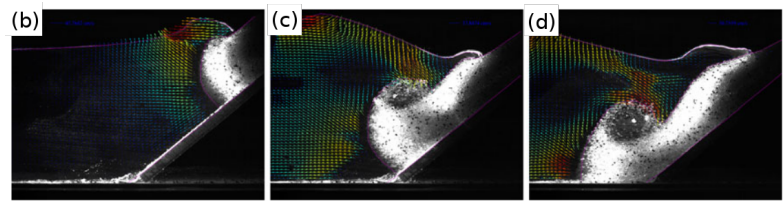

Figure 2: Subaerial test case. (a) Sketch of the experimental set-up and (b)-(d) snapshots of the generation area at different times $(0.2 \mathrm{~s}$ between pictures) for a test involving a slide mass of $3 \mathrm{~kg}$, from Viroulet et al. (2013).

A $t=0 \mathrm{~s}$, the granular material is released. The glass beads slide down the slope toward the bottom of the tank and finally stop under the action of the different dissipative forces (Figure 2(b)-(d)). When the slide penetrates into water, the free surface is lifted up and forms the first wave that propagates in the tank followed by trailing waves in which the second one appears to be the largest. 
Case 2 : Submarine case In the submarine case (Grilli et al., 2017), a granular media, whose initial shape is similar to the subaerial case, is released underwater. A sketch of the $6.27 \mathrm{~m}$ long flume is presented in Figure 3 (a). A $2 \mathrm{~kg}$ mass of glass beads of diameter $4 \mathrm{~mm}$ and density of $2500 \mathrm{~kg} . \mathrm{m}^{-3}$ is released on a slope of $35^{\circ}$ (Figure $3(\mathrm{~b})-(\mathrm{g})$ ). The water depth is $0.330 \mathrm{~m}$. Four gauges are placed at $600,1600,2600,3600 \mathrm{~mm}$ from the gate to record the water surface fluctuations. Two larger waves followed by a wave train are generated. The wave is smaller than that of the subaerial case (maximum elevation for the subaerial case: $2 \mathrm{~cm}$, and for the submarine case: $0.6 \mathrm{~cm}$ ). Moreover, contrary to the studied subaerial case, the second wave is larger than the first one.

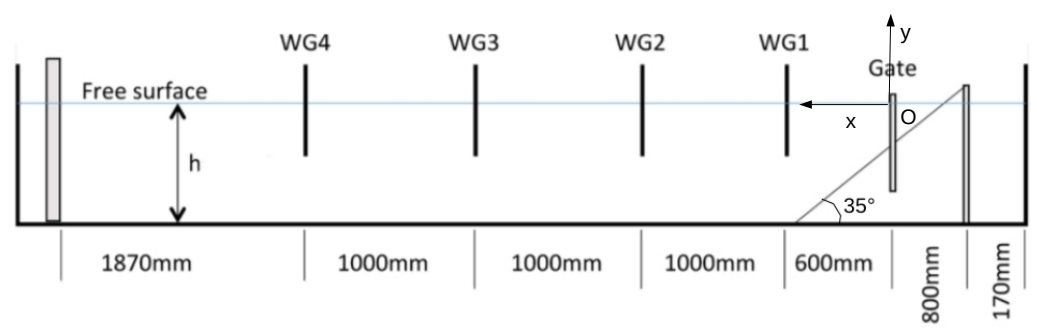

(a)

(b)
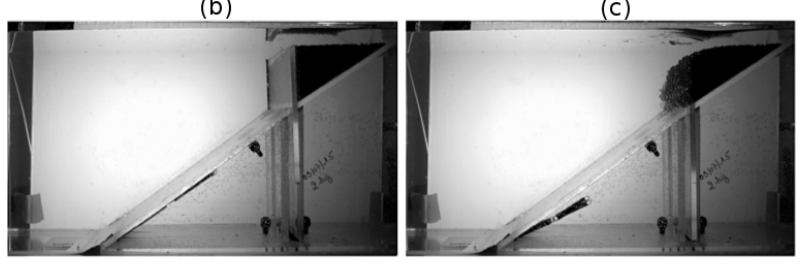

(d)
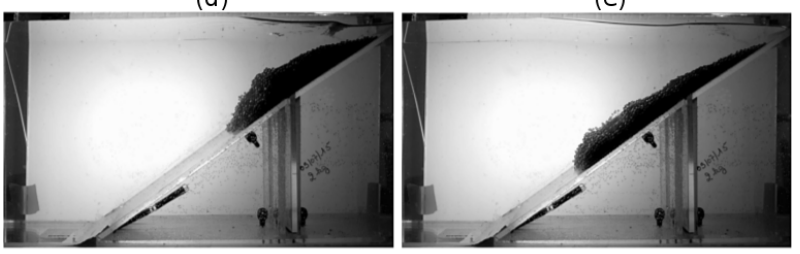

(f)

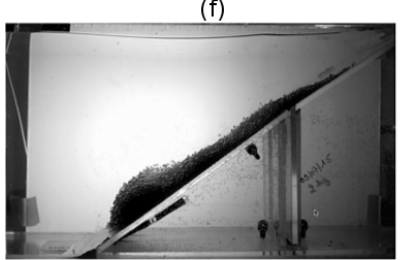

(g)

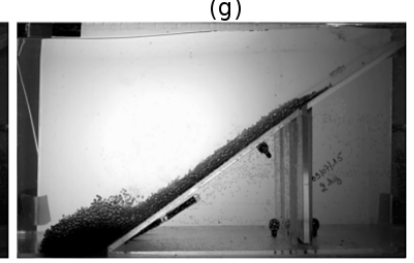

Figure 3: Submarine landslide test case. (a) Sketch of the experimental set-up and (b)-(g) snapshots of the generation area at different times (i.e., photographs (b) to (g)) $t=-0.125,0.02,0.17,0.32,0.47,0.62 s$, from Grilli et al. (2017).

\subsubsection{Subaerial landslide simulation results}

As previously mentioned, the slide is modelled as a Newtonian fluid. The idea is to use the fluid viscosity as a free parameter in order to approach a granular behaviour. This is supported by the $\mu(I)$ formulation for granular medium, which uses a variable viscosity in space and time in conjunction with a flow threshold. Here, the idea is to use a constant value as a first approximation and to neglect the threshold whose effect is supposed to be weak during the wave generation process. The slide density value to be taken in the simulation may be questioned because the fluid between the beads and the slide volume vary during the experiment (Figure 2). At $t=0 \mathrm{~s}$, when the slide is at rest above water with a random close pack which characterizes the maximum volume fraction of randomly packed solid bodies, the maximum volume fraction of the beads is about $60 \%$, which gives a density of $1500 \mathrm{~kg} \cdot \mathrm{m}^{-3}$ for the slide. However, the slide is 
moving beneath the water with a maximum density of $1900 \mathrm{~kg} \cdot \mathrm{m}^{-3}$ for most of the experiment. Moreover, during the experiment, the slide volume expands (almost a 50\% expansion) as the beads does not form a close pack (Viroulet, 2013; Viroulet et al., 2013). For this reason, the latter density is maximal and simulations were run with this density to show its influence on the wave heights. The change of density was found to have a little influence on the wave heights. First and second waves are only slightly higher with the $1900 \mathrm{~kg} \cdot \mathrm{m}^{-3}$ density. Therefore, in the present simulations, the density is set to $1500 \mathrm{~kg} \cdot \mathrm{m}^{-3}$.

The focus is now on the influence of the viscosity value. With a low viscosity, the slide moves faster and a bulge shape slide front is observed similar to the experimental results (Figure 4(a)). The best results in terms of the slide motion are obtained with a viscosity of $2 \mathrm{~Pa} . \mathrm{s}$, even though in this case the slide is a bit slower than in the experiment. However, the height of the first wave at the four gauges (Figure 4(b)) appears to be almost twice as high as the experimental results for this viscosity. At a lower viscosity, the water free surface elevation seems to be discontinuous because it is more perturbed due to wave breaking and harder to capture at the gauges. The first wave and the wave train are well reproduced for a viscosity of 10 Pa.s, even though the slide at this viscosity is shown to be slower than in the experiment. The same overall behaviour was observed in the second test performed in Viroulet et al. (2014) with a glass bead diameter of $10 \mathrm{~mm}$ (results not shown here), but a higher value of viscosity has to be set in order to fit the experimental wave heights.

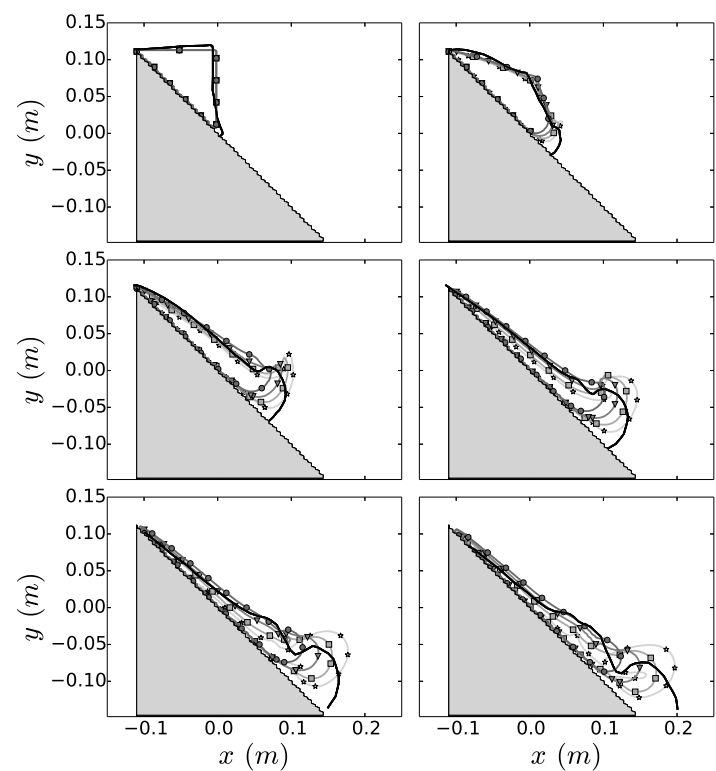

(a)
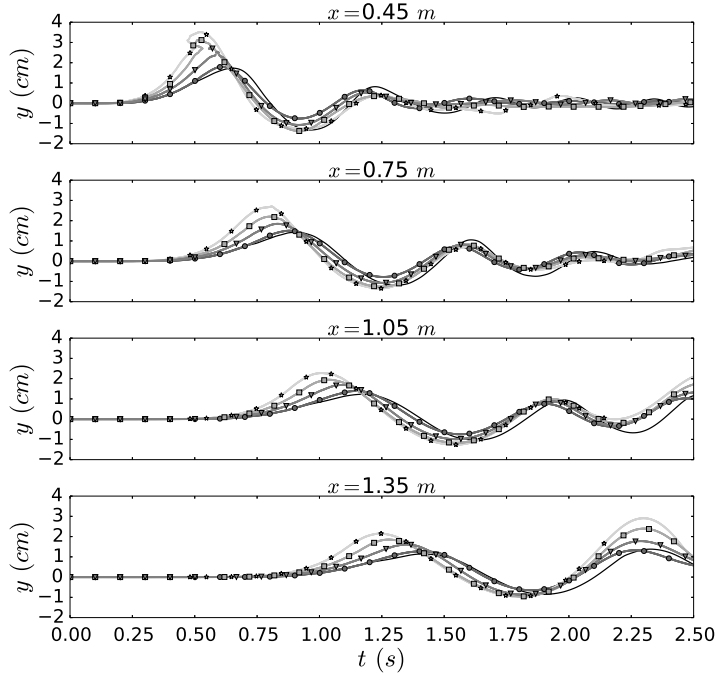

(b)

Figure 4: Simulation results for different values of the slide viscosity (Newtonian fluid) on the subaerial case. (a) snapshots of the slide contours with first panel $t=0 s$ and $0.1 s$ time difference between figures, and (b) temporal changes of the water free surface elevation at the experimental gauges. The experimental results (black line) in comparison with the simulation results for different viscosity values, $\eta=1 P a . s$ (stars), $\eta=2$ Pa.s (squares), $\eta=5$ Pa.s (triangles) and $\eta=10$ Pa.s (circles).

Based on the $\mu(I)$-rheology, Ionescu et al. (2015) proposed the following equation to have an idea of the viscosity of the equivalent fluid generated during the initial phase of sliding:

$$
\eta=\left(\mu_{s}+\frac{\Delta \mu}{\frac{I_{0} \sqrt{p / \rho_{g}}}{d \sqrt{2} D_{2}}+1}\right) \frac{p}{\sqrt{2} D_{2}}
$$

In the present experiment, the following parameter values can be considered: $p=\rho_{s} g \frac{h_{0}}{2}, D_{2}=\frac{\sqrt{g h_{0}}}{h_{0} / 2}$, $d=0.0015 \mathrm{~m}, h_{0}=0.11 \mathrm{~m}, \rho_{g}=2500 \mathrm{~kg} \cdot \mathrm{m}^{-3}$ and $\rho_{s}=1500 \mathrm{~kg} \cdot \mathrm{m}^{-3}$. As for the $\mu(I)$ parameters, they are set to $I_{0}=0.279, \mu_{s}=0.38$ and $\Delta \mu=0.26$ following Pouliquen and Forterre (2002) where the beads used in their experiments are similar in size and material. This leads to an "average" viscosity 
$\eta=12.8$ Pa.s. The latter value is close to the one finally chosen after test and error procedure for the Newtonian fluid consideration.

Simulations were also performed on the subaerial case with the $\mu(I)$-rheology implemented in THETIS for which the parameters are defined by the granular media. The results of these simulations have to be considered with caution as the $\mu(I)$ model is only supposed to be valid in a dry granular flow. This simulation is therefore here mainly to show the viscosity which develop in the slide at the very first instant of the simulation. This is relevant as the slide should not be drastically influenced by water in the first instant of the process and we shall show later that this stage is critical in terms of wave generation. Figure 5 shows the evolution of the viscosity inside the slide during the simulation. After entering the water, the viscosity at the front part of the slide gets higher and the slide quickly stops due to the non physical influence of the water hydrostatic pressure in the $\mu(I)$ law. However, waves are generated very quickly in this experiment and we can see in Figure 6 (b) that the wave height is quite close to the experimental results. Compared to the computation with the Newtonian fluid, during the first $0.5 \mathrm{~s}$ where the waves are generated, the deformation of the slide is very similar to the Newtonian slide (Figure 6 (a)) illustrating the fact that the viscosity computed in the $\mu(I)$ model is close to the one chosen in the Newtonian model.
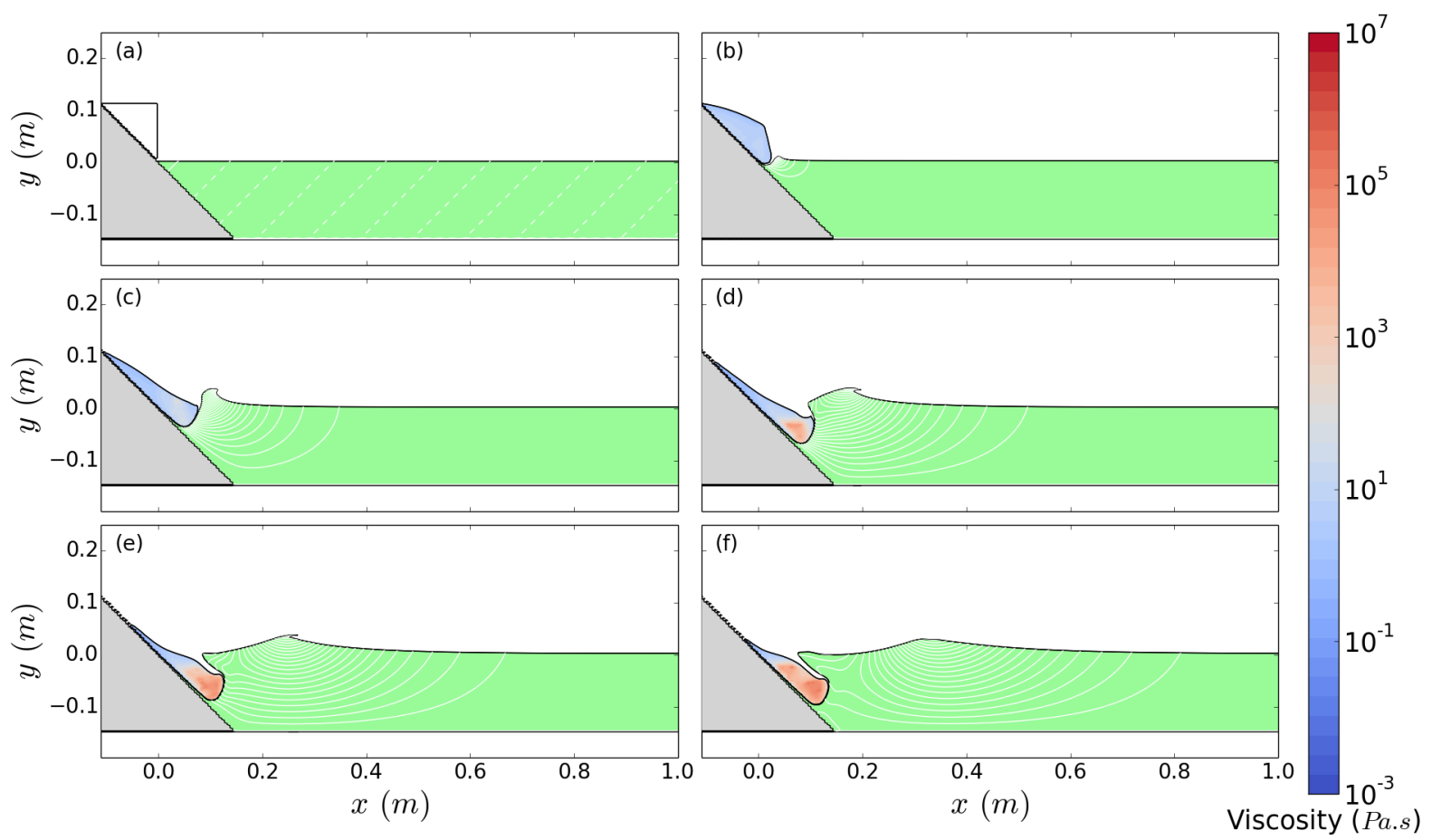

Figure 5: Simulation results for the $\mu(I)$ rheology on the subaerial case. Evolution of the viscosity inside the slide and water flow streamlines, first panel $t=0 \mathrm{~s}, 0.1 \mathrm{~s}$ time difference between figures.

\subsubsection{Submarine landslide simulation results}

For the submarine landslide case, the numerical domain has a length of $6.27 \mathrm{~m}$ and a water depth of $0.33 \mathrm{~m}$. The slide is modelled as a Newtonian fluid, with parameters defined in Grilli et al. (2017), i.e. a viscosity of 0.01 Pa.s and a density of $1951 \mathrm{~kg} \cdot \mathrm{m}^{-3}$. A few other viscosity values are also considered to evaluate the sensitivity of the model to this parameter.

Results of the slide shapes and the wave heights are presented in Figures 7 and 8 . The slide shape evolution as well as the slide overall velocity is shown to strongly depend on the viscosity value. For the low viscosity used in Grilli et al. (2017), the slide front exhibits a bulge head due to the presence of a strong counter-clockwise vortex generated at the water/slide interface. It is probable that the model used in Grilli et al. (2017) could not observe this feature due to its formulation, but it has a strong influence on the overall slide velocity. This vortex gets weaker and weaker with increasing the viscosity 

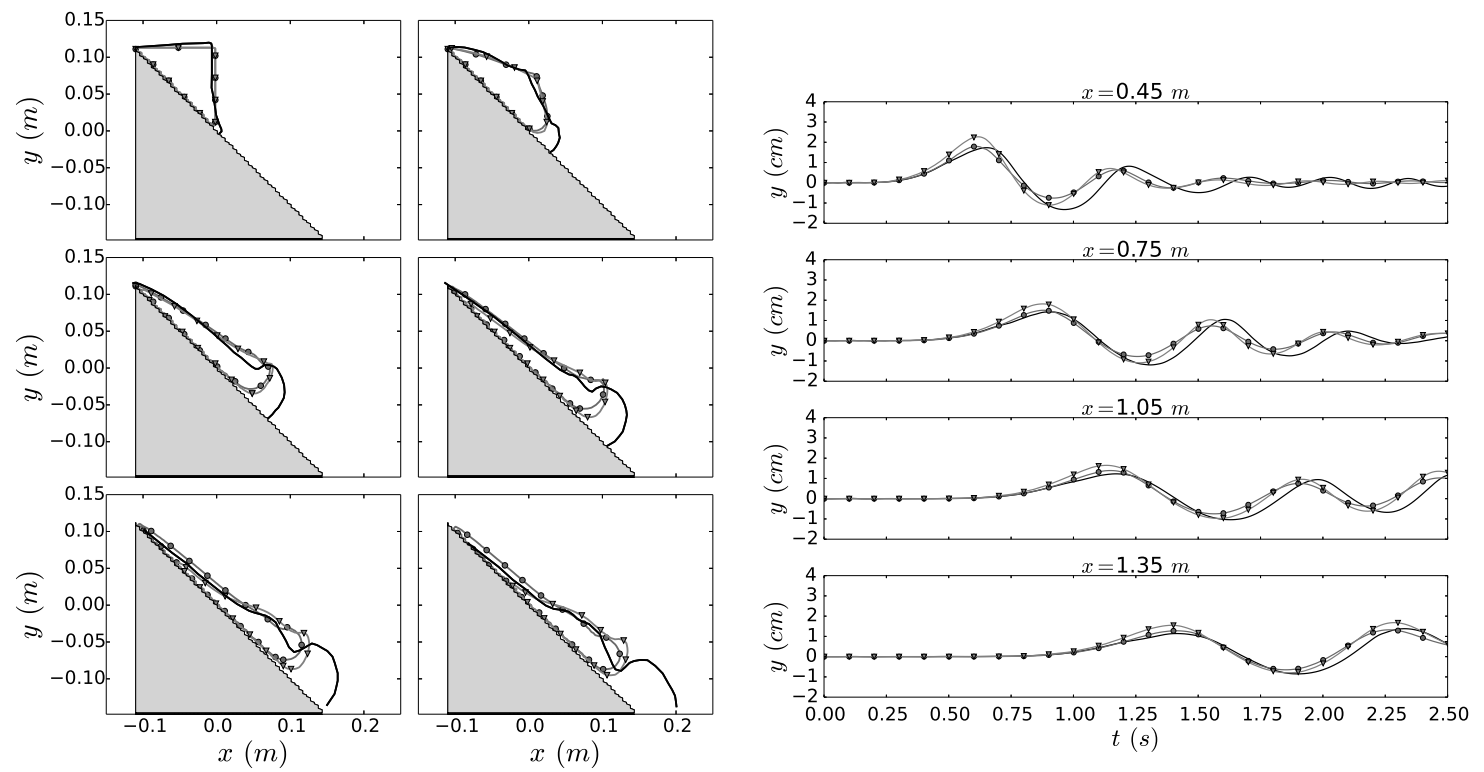

Figure 6: Simulation results for the $\mu(I)$ rheology on the subaerial case. (a) snapshots of the slide contours and (b) temporal changes of the water free surface elevation at the experimental gauges. The experimental results (black line) in comparison with the THETIS simulation results, Newtonian fluid $\eta=10$ Pa.s (circles) and $\mu(I)$-rheology (triangles). First panel $t=0 \mathrm{~s}, 0.1 \mathrm{~s}$ time difference between figures.

value. The experimental snapshots also show that the occurrence of this vortex are more or less consistent with the results corresponding to the two stronger viscosities tested in our simulations. Nevertheless, in all the cases tested, the slide motion appears to be very slow compared to the experiment. The best approximation would be with the lowest viscosity but due to the aforementioned large vortex and the subsequent additional drag induced, the slide velocity cannot reach higher values.

Figure 8 shows that with a slide viscosity of 0.01 Pa.s, the first wave is higher than the experimental value and the wave train is not correctly reproduced on the first gauge. By increasing the viscosity, the generated waves are lower. We observe that with a viscosity of 1 Pa.s, the first wave is close to the experimental results as well as the first waves in the wave train. Generally, the wave heights are closer to the experiment when the numerical slide moves slower than the experimental slide with having a higher viscosity.

\subsection{Energy transfers}

Simulations are now interpreted in terms of energy transfer from the slide to the generated waves. The results are shown with a dimensionless form of the time $t^{*}=t \sqrt{g / h}$.

\subsubsection{Subaerial test case}

The total energy of the system \{air+water+slide\} should be constant in time. Figure 9 (a) illustrates the time evolution of the relative error of the total system energy which can be attributed to numerical dissipation during simulations with the slide modelled as a Newtonian fluid in the subaerial case. This dissipation is very low and reaches only $0.3 \%$ at $2 s\left(t^{*}=16.3\right)$, the time at which, as will be shown below, most of the energy has been transferred. Therefore, the total energy is properly conserved during the simulation. Additionally, the air total energy varies only very slightly. Accordingly, only the transfer of energy between the water and the slide is considered in these simulations.

Figure 9 (b) illustrates the time evolution of energy components and energy dissipated at time $t$ in the slide, with $E_{0}$ as the initial slide potential energy (also equal to the slide initial mechanical energy) taking the tank bottom as reference. As the gate opens, the slide is released and flows over the slope. Its velocity increases as well as its kinetic energy (in red) but with a fairly small amount compared to the 

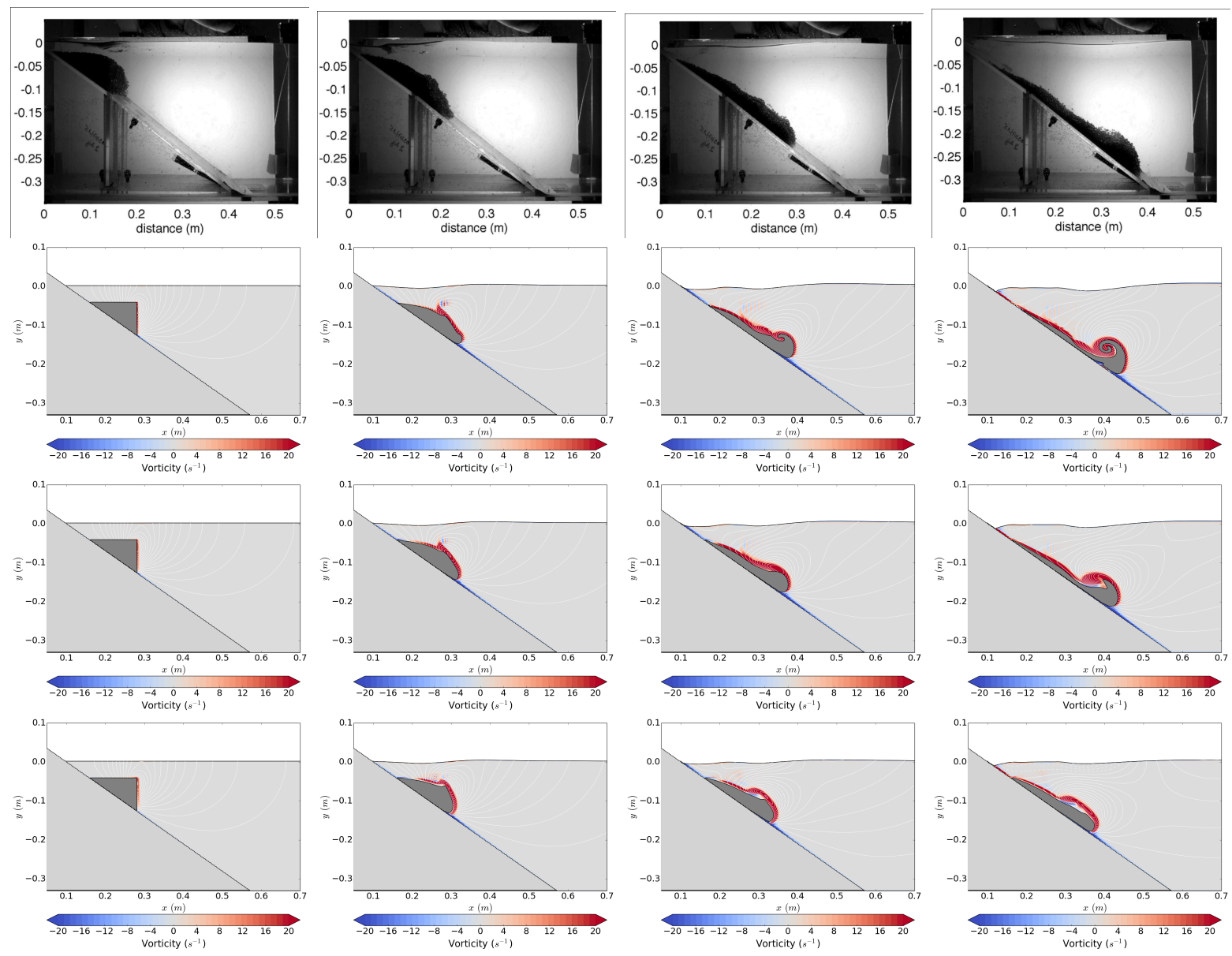

Figure 7: Slide colour function and vorticity contours for the submarine landslide benchmark (Grilli et al., 2017) with the experimental results in the first row and simulation results (following rows) for different values of viscosity $(\eta=0.01,1,10$ Pa.s for second, third and fourth row respectively $)$ at times $t=0.02,0.17,0.32,0.47 s$.

potential energy decrease. Conversely, the total viscous dissipation, linked to the slide velocity and the viscosity value, increases very significantly and reaches an asymptotic value as the slide stops. Likewise, the energy transferred to the water appears very significant (similarly as the dissipation) compared to the slide kinetic energy.

Water is set in motion by the slide during the penetration stage and the free surface deforms inducing kinetic and potential energies increase. The viscous dissipation in water (not shown here) is very small because of the low viscosity of water and is of the order of $0.1 \%$ of the initial total water energy at the end of the simulation.

Regarding the distinction between wave generation and propagation, in this subaerial case, vorticity is confined near the slide and at the free surface (Figure 10). At the right of the slide, we observe the typical stream contours of a wave field. The limit between both zones, as defined previously, is therefore relevant. In the propagation zone, wave energy is computed. We see (Figure 9 (c)) that the wave kinetic and potential energy increases quickly at the beginning of the simulation and stabilizes around $t=0.5 \mathrm{~s}$ $\left(t^{*}=4\right)$. The significant variation of energy around $t=1.5 \mathrm{~s}\left(t^{*}=12.2\right)$ is explained by the reflection of the first wave on the downwave tank end (Figure $9(\mathrm{~d})$ ).

Figure 9 (e) shows the wave energy divided by the slide initial energy. The wave energy reaches its maximum at $t=0.45 \mathrm{~s}\left(t^{*}=3.7\right)$ and then decreases slightly before stabilizing. This reduction of energy is due to the slight breaking of the first wave which may be observed on the third snapshot of Figure 10. Therefore, we can conclude that the energy transfer to the wave field is very quick in this case and nothing more happens in terms of energy transfer to the free surface downstream the slide after $t=0.45 s\left(t^{*}=3.7\right)$. 

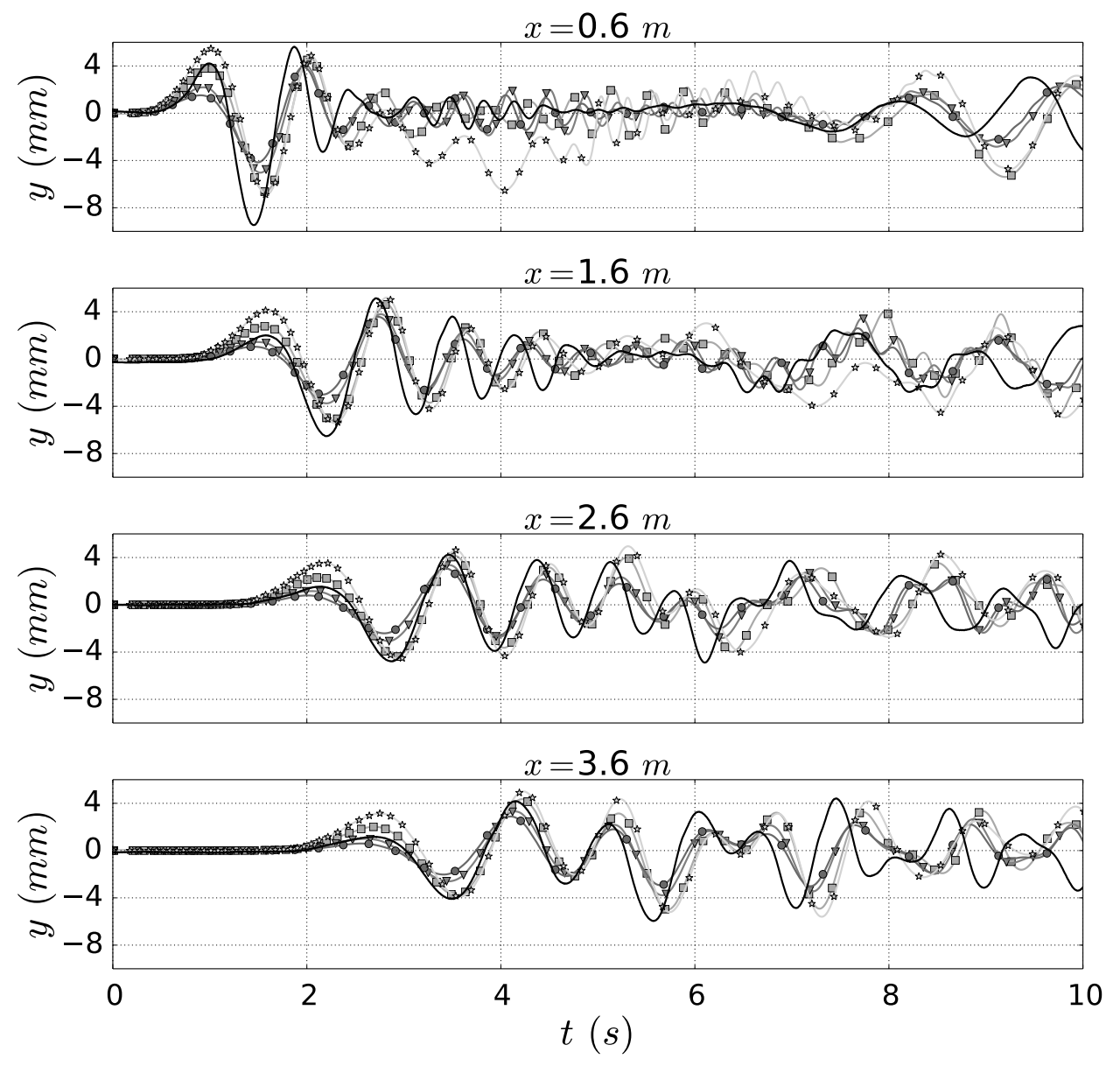

Figure 8: Elevation of the water free surface for the submarine landslide benchmark (Grilli et al., 2017), comparison between experiment (black) and simulation results with different viscosity values (0.01 Pa.s: stars, 1 Pa.s: squares, 5 Pa.s: triangles, 10 Pa.s: circles).

The energy transfer ratio is of course different considering equation 17 or equation 18 , the former equation does not consider the part of the slide energy which transfers to kinetic energy and dissipation. These transfer ratios are plotted on Figure 9 (e). Note that for the first instants of energy transfers from slide to water, the transfer ratio involves very small numbers whose combination appears to stabilize only after a small time duration. Therefore the resulting ratio is only plotted after this duration which varies from one case to the other one. Logically, the energy transfer ratio given by equation 17 has a lower value all along the simulation.

The energy transfer ratio from slide into waves shows a peak at $t=0.2 \mathrm{~s}\left(t^{*}=1.6\right)$ of around 0.5. When all the energy has been transferred to waves (i.e., $\left.t=0.45 \mathrm{~s}\left(t^{*}=3.6\right)\right)$, the energy transfer ratio is about one third evidencing the efficiency of this subaerial slide to produce energetic waves. Afterwards, no more energy is transferred to the surface (and propagating off-shore) but as energy is still transferred to the water mass (Figure 9 (b)), the energy ratio decreases to an asymptotic value around 0.13 (0.067 with the energy transfer ratio of Jiang and LeBlond (1992)).

\subsubsection{Submarine test case}

Results for the submarine case are presented in Figure 11. The energy transfer is very low, which explains the small waves observed in this case, compared to the studied subaerial case.

As in the submarine case, the total energy of the system $\{$ air+water+slide $\}$ is preserved during the simulation with an error of about $0.012 \%$ at $t=5 s\left(t^{*}=27.3\right)$ (Figure 11 (a)). 
Figure 11 (b) illustrates the repartition of energy in the slide during the simulation. In the submarine case, the initial slide potential energy $E_{0}$ is larger than in the previous subaerial case $(24.8 J$ and $19.7 J$, respectively) due to a larger initial height. Therefore, and because the subaerial case starts close to the free surface with a nil initial velocity, a larger fraction (i.e., 0.6) is in overall transferred to water in the submarine case compared to the subaerial case. Nevertheless, as we shall see in the next paragraph, this does not mean that the transfer to waves is larger.

Similar to the studied subaerial case, the wave field potential energy (Figure 11) increases and then stabilizes itself to a constant value. However, in this case, the kinetic energy does not follow this pattern. It first increases faster than in the previous case, where the kinetic energy followed the potential energy (Figure $9(\mathrm{c})$ ). This behaviour is explained by the recirculation of the flow above the slide illustrated in Figure 13. When the slide is flowing over the slope, significant velocities appear just above the slide front. However, as soon as the slide reaches the bottom, this recirculation quickly moves to the back of the slide explaining the fast decrease of the kinetic energy in the propagation zone from the time $t=0.9 \mathrm{~s}\left(t^{*}=4.9\right)$. It then decreases to reach the potential energy value but subsequently, increases again around $t=2 \mathrm{~s}\left(t^{*}=10.9\right)$. This unexpected behaviour is explained by the presence of strong vorticity in the propagation zone (Figure 12). This vorticity, which was generated by the slide front, is then advected more rapidly than the slide itself. Therefore, the mechanical wave energy will be taken as twice the potential energy even if this hypothesis is known to be only approximative (Fritz et al., 2004; Heller et al., 2016).

In this submarine case, the slide kinetic energy and the dissipation have low values compared to the slide potential energy so the transferred energy is almost equal to the potential energy (Figure 11 (a)). The difference between the ratio introduced by Jiang and LeBlond (1992) and Equation (18) is not very marked with a final transferred energy ratio of about 0.01 with the wave mechanical energy taken as twice the potential energy until the waves are reflected on the opposite side of the tank (Figure $11(\mathrm{~d})$ ). Moreover, the efficiency of the system, when no more energy is transferred to the waves (at $t=1 \mathrm{~s}$, $\left.\left(t^{*}=5.46\right)\right)$, is around 0.03, namely one order of magnitude less than the studied subaerial case.

\section{Discussion}

The simulations performed in this paper are based on a simple Newtonian rheology with only one parameter, the viscosity, which is here used as a free parameter to reproduce coarsely a granular behaviour. The value of the viscosity found by fitting the waves with the subaerial experiment was found to be consistent to what should be expected in average from a $\mu(I)$ granular rheology. Nevertheless, for both models, even if similar waves are generated, the slide shape and velocity are quite different.

Several strategies have been tried to produce faster slides, such as vanishing the basal friction, tuning the density, etc., but they did not have any significant effect on the slide velocity. The reason may be that the slide is simulated as an equivalent phase instead of a granular water mixed medium. The difference is important as the latter allows water to flow within the granular medium, whereas the former does not. This difference may significantly reduce the drag force on the slide, which defines the value of the slide final velocity. A second approximation is that the volume fraction of grain is constant in the simulations, whereas, looking at the experiment, the granular slide expands significantly during the sliding and the volume fraction decreases.

The energy transfer ratio is often based on the potential energy released by the landslides (Jiang and LeBlond, 1992) or the slide kinetic energy (Fritz et al., 2004). The present paper shows that the slide energy dissipation should also be taken into account to better understand the energy transfer process. The asymptotic transfer ratio for the studied subaerial case is twice its value as compared to the cases without taking the dissipation and kinetic energy into account. However, it has been highlighted, in section 3.2.2, that in the submarine case, the potential energy is predominant over the dissipation and kinetic energy. In this case, the two energy transfer ratios give similar values.

Comparing energy transfer values between different studies may not be completely relevant as the references or energy considered are often not the same in all studies. For instance, Watts (1998) and Ataie-Ashtiani and Nik-Khah (2008) used a slide energy based on the terminal velocity of the submarine landslide or Jiang and LeBlond (1992) calculated the slide potential energy with a reference at the still water level whereas the present study used a slide potential energy based on the bottom level.

In this work, in the subaerial case, about $60 \%$ of the available potential energy is released during the 
simulation. Slide energy dissipation accounts for about $45 \%$ of this value whereas $50 \%$ is transferred to water and $6-7 \%$ to waves. This value drops to $4 \%$ when it is expressed in terms of $E_{0}$. The latter values are at least consistent with the results presented for instance in Yavari-Ramshe and Ataie-Ashtiani (2019) where conversion ratios for subaerial slides were in the range 5-15\% and logical if we consider that the case studied involved a nil initial slide velocity.

In the submarine case (Grilli et al., 2017), $90 \%$ of the available potential energy is released during the simulation. About $25 \%$ of this energy is dissipated in the slide, $75 \%$ is transferred to water and $1.4 \%$ to waves $\left(1.2 \%\right.$ when expressed in terms of $\left.E_{0}\right)$. Comparing to other experimental results, this latter value is in agreement with the range of 1-7.5\% found by Yavari-Ramshe and Ataie-Ashtiani (2019) for submarine slides energy conversion ratios. It should be remarked that the final energy conversion ratio is not completely sufficient to define the efficiency of the process. For instance the low value of $4 \%$ obtained in the subaerial case studied actually hides a very quick energy transfer during which the efficient energy transfer to waves is $30 \%$ in average.

The energy transfer to the free surface was indeed shown to be very quick. For instance, in the studied subaerial case, the whole wave field seems completely determined at $t \approx 0.5 \mathrm{~s}$. This confirms the findings of Heller et al. (2016) who, based on very detailed measurements providing novel instantaneous slide-water interaction power graphs, showed that the majority of the energy, in their experiment involving a solid subaerial slide, was transferred within $0.5 \mathrm{~s}$.

In our study, this time corresponds to the situation shown in Figure $5((\mathrm{~d})-(\mathrm{e}))$. This tend to show that the trailing waves may not be generated by the slide. In order to evidence this further, a simulation was carried out with a slide artificially forced to stop very early in the simulation of the subaerial case (stop time $t_{s}$ respectively : $\left.t=0.2,0.3,0.4,0.5,0.6,0.7 \mathrm{~s}\left(t^{*}=1.6,2.4,3.3,4.1,4.9,5.7\right)\right)$ by using penalizing techniques (Ducassou et al., 2017).

For the lowest stop time value (i.e., $t_{s}=0.2 s\left(t^{*}=1.6\right)$ ), the first wave is noticeably smaller than measured (Figure 14). With $t_{s}=0.3 \mathrm{~s}\left(t^{*}=2.4\right)$, the wave train is only very slightly smaller than in the experiment and is very well reproduced for larger values of $t_{s}$. Figure 15 (a) illustrates the energy transferred by the slide to water in each simulation. A drop is observed in the wave total energy (Figure 15 (b)) due to a very quick water slowdown around the slide, but not at the free surface. This is less noticeable as the stop time is high and the flow around the slide exhibits lower velocities. It can be clearly observed (Figure $15(\mathrm{~b}))$ that the energy released by the slide after $t=0.5 s\left(t^{*}=4.1\right)$ does not contribute significantly to the wave energy, its evolution in time being the same as the one of the slide stopped at $t=0.5 s\left(t^{*}=4.1\right)$ or after.

As a wave train is observed in each simulation even with the slide stopped very early, this support the hypothesis that the wave train is not generated by energy transfer from the slide. It is still not clear in the simulations whether the second wave is generated by the reflection on the slope or by dispersion of the first wave. But the wave train following the second wave is clearly generated by dispersion of the latter.

If we focus now on the first wave, the simulations with the slide stopped demonstrated that the wave can be quite accurately simulated without modelling the entire slide process (i.e. from the release to the deposition). The slide dynamics and shapes are quite different considering the Newtonian simulations (with the slide stopped or not) and the experiment, however, they succeed in generating very similar waves. This emphasizes the importance of landslide parameters for the accurate generation of waves.

The same procedure is reproduced for the submarine slide case in Figure 16. As in the subaerial case, the wave field is correctly reproduced when artificially stopping the slide at different times around the assumed end of slide energy transfer to the free surface. This again demonstrate that the wave field is completely determined as soon as the transfer to the surface is over which occurs within $1 s$ even in this submarine case. Nevertheless, this procedure of stopping the slide is not completely harmless as the slide acts then as a rigid obstacle which can influence the free surface in certain conditions.

The two cases studied in this paper are different in initial conditions, channel width, and bottom slope. In order to have more comparable cases, a new simulation was performed involving a Newtonian slide with viscosity $\eta=10$ Pa.s in Viroulet et al. (2013)'s configuration but using the same initial submergence as in Grilli et al. (2017). The energy transfer plots (Figure 17) show a very quick energy transfer to the free surface in less than $0.25 \mathrm{~s}\left(t^{*}=2\right)$ and the energy transfer is a little less than $1 \%$ of the reference energy compared to the $4 \%$ found in the corresponding subaerial case.

Finally, in this paper, all the cases studied showed a very quick energy transfer. The reasons explaining this observation are likely: 1- regarding the subaerial slide, the nil initial velocity. 2- for both subaerial 
and submarine cases, the relatively large slope which causes a quick increase in the local depth above the slide.

In analysing the energy transfer, a relevant parameter is the local slide Froude number defined as the ratio of the instantaneous slide velocity and the local wave celerity $(\sqrt{g h})$ where $h$ is the local depth. The energy transfer is maximum around $F r=1$ (Pelinovsky and Poplavsky, 1996). For instance, regarding the subaerial case, the energy transfer is expected to be larger and longer for large impact velocities when reaching the water line. And conversely, steep slopes allow waves to quickly move away from the slide influence area.

\section{Conclusions}

Navier-Stokes simulations of landslide modelled as a Newtonian fluid have been presented for three cases: subaerial and submarine granular landslides previously studied experimentally and one synthetic submarine slide case. By comparing with experimental results, we showed that the waves can be accurately reproduced using a simple Newtonian slide model with adjusting the viscosity. We also showed that the viscosity value found is consistent with the $\mu(I)$ granular law in the subaerial case. Even though the wave field obtained is satisfactory, the slide motion, in the two main cases, are significantly slower than in the experiment. This illustrates the need for a two phase flow formulation for the slide which allows a differential flow between water and grain.

Energy transfers from the slide to the wave field have also been studied, leading to the following conclusions:

- Waves gain all their energy in a relatively short period in all the cases simulated. This observation may be related to the initial nil slide velocity (subaerial case) and the relatively large slope values considered (both cases).

- The process is significantly more efficient in the subaerial case than in the submarine case. During the wave generation process, about one third of the slide energy conveyed to water during the slide process is transferred to wave energy. This value is 10 times less in the submarine case.

- The simulation results showed a significant energy dissipation within the slide for both subaerial and submarine cases.

- Finally, by a mandatory stop of the slide on different distances along the slope, it is shown that the wave train is more a consequence of the first wave than a consequence of energy transfer. Therefore, for slide configurations comparable to the ones studied in this paper, a complete description of the slide dynamics until run-out may not be necessary to capture the generated waves.

\section{Acknowledgement}

This work was funded by the FP7 EU research program ASTARTE (Grant No.: 603839), the PIA RSNR French program TANDEM (Grant No.: ANR-11-RSNR-00023-01) and the ANR RAVEX (ANR-16-CE030002) project. Financial support from the French Ministry of Higher Education, Research and Innovation for the PhD fellowship of Lucie Clous is gratefully acknowledged.

\section{Nomenclature}

$\begin{array}{ll}\Delta x & \text { Horizontal length interval }[\mathrm{L}] \\ \Delta y & \text { Vertical length interval }[\mathrm{L}] \\ \eta & \text { Fluid dynamic viscosity }\left[\mathrm{ML}^{-1} \mathrm{~T}^{-1}\right] \\ \lambda & \text { Energy transfer ratio } \\ \mu & \text { Friction coefficient }\end{array}$




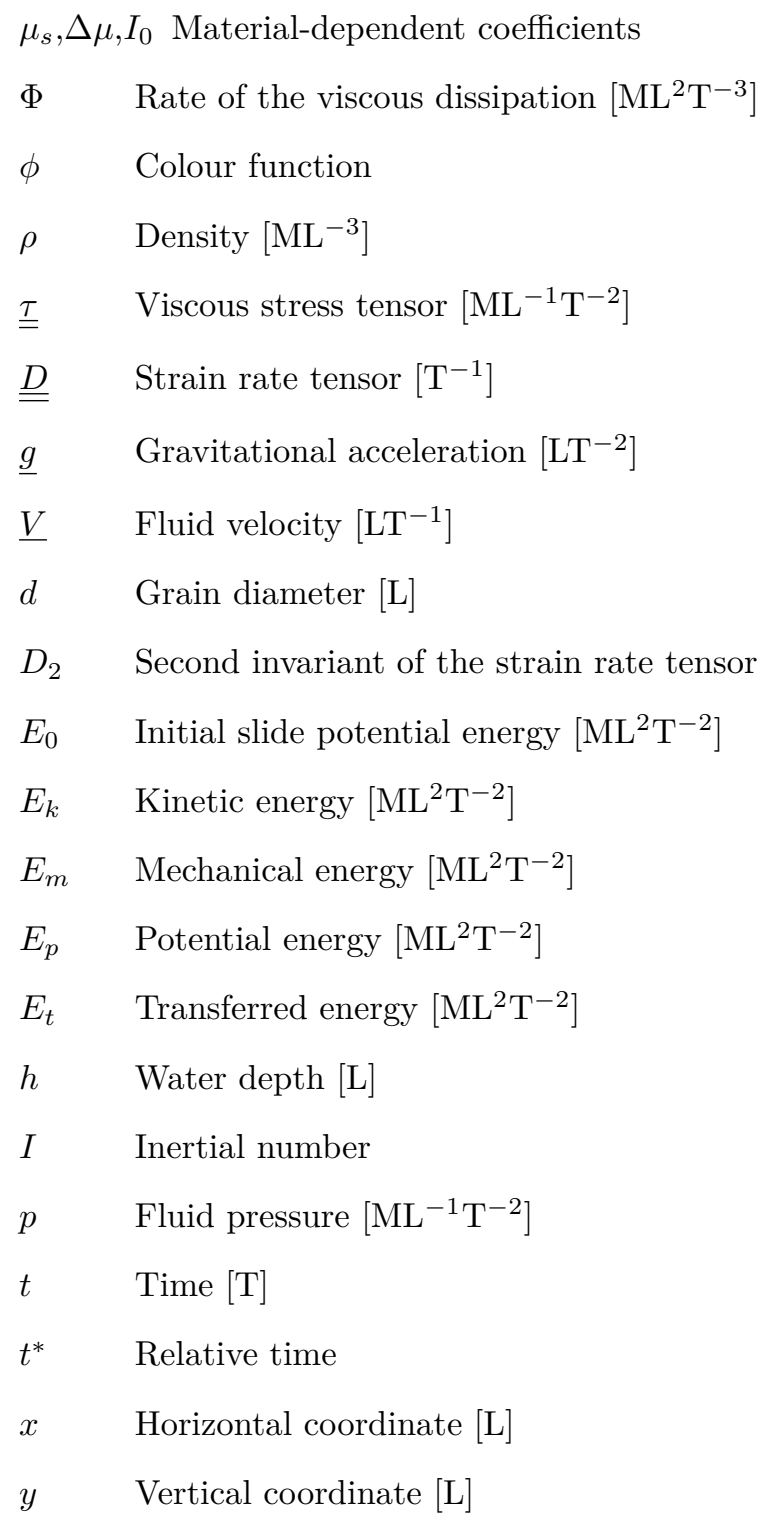

\section{References}

Abadie S, Morichon D, Grilli S, Glockner S (2010) Numerical simulation of waves generated by landslides using a multiple-fluid Navier-Stokes model. Coastal Engineering 57(9):779-794, DOI 10.1016/j.coastaleng.2010.03.003

Abadie SM, Harris JC, Grilli ST, Fabre R (2012) Numerical modeling of tsunami waves generated by the flank collapse of the Cumbre Vieja Volcano (La Palma, Canary Islands): Tsunami source and near field effects. Journal of Geophysical Research 117(C5), DOI 10.1029/2011JC007646

Ataie-Ashtiani B, Najafi-Jilani A (2008) Laboratory investigations on impulsive waves caused by underwater landslide. Coastal Engineering 55(12):989-1004

Ataie-Ashtiani B, Nik-Khah A (2008) Impulsive waves caused by subaerial landslides. Environmental Fluid Mechanics 8(3):263-280, DOI 10.1007/s10652-008-9074-7

Cassar C, Nicolas M, Pouliquen O (2005) Submarine granular flows down inclined planes. Physics of Fluids 17(10):103301, DOI 10.1063/1.2069864

Chassaing P (2000) Mécanique des fluides. Eléments d'un premier parcours Cépaduès éditions 
Courant R, Friedrichs K, Lewy H (1967) On the partial difference equations of mathematical physics. IBM Journal of Research and Development 11(2):215-234

Desombre J, Morichon D, Mory M (2012) Simultaneous surface and subsurface air and water flows modelling in the swash zone. Coastal Engineering Proceedings 1(33):56

Ducassou B, Nuñez J, Cruchaga M, Abadie S (2017) A fictitious domain approach based on a viscosity penalty method to simulate wave/structure interaction. Journal of Hydraulic Research 55(6):847-862

Fritz HM, Hager WH, Minor HE (2004) Near field characteristics of landslide generated impulse waves. Journal of Waterway, Port, Coastal, and Ocean Engineering 130(6):287-302, DOI 10.1061/(ASCE) 0733-950X(2004)130:6(287)

GDR MiDi (2004) On dense granular flows. The European Physical Journal E 14(4):341-365, DOI 10.1140/epje/i2003-10153-0

Goda K (1979) A multistep technique with implicit difference schemes for calculating two- or threedimensional cavity flows. Journal of Computational Physics 30(1):76-95, DOI 10.1016/0021-9991(79) 90088-3

Grilli ST, Shelby M, Kimmoun O, Dupont G, Nicolsky D, Ma G, Kirby JT, Shi F (2017) Modeling coastal tsunami hazard from submarine mass failures: effect of slide rheology, experimental validation, and case studies off the US East Coast. Natural Hazards 86(1):353-391

Heinrich P (1992) Nonlinear water waves generated by submarine and aerial landslides. Journal of Waterway, Port, Coastal, and Ocean Engineering 118(3):249-266

Heller V, Hager W (2011) Wave types of landslide generated impulse waves. Ocean Engineering 38(4):630640

Heller V, Hager WH (2010) Impulse product parameter in landslide generated impulse waves. Journal of Waterway, Port, Coastal, and Ocean Engineering 136(3):145-155, DOI 10.1061/(ASCE)WW. 1943-5460.0000037

Heller V, Bruggemann M, Spinneken J, Rogers BD (2016) Composite modelling of subaerial landslidetsunamis in different water body geometries and novel insight into slide and wave kinematics. Coastal Engineering 109:20-41

Ionescu IR, Mangeney A, Bouchut F, Roche O (2015) Viscoplastic modeling of granular column collapse with pressure-dependent rheology. Journal of Non-Newtonian Fluid Mechanics 219:1-18, DOI 10.1016/ j.jnnfm.2015.02.006

Jiang L, LeBlond PH (1992) The coupling of a submarine slide and the surface waves which it generates. Journal of Geophysical Research 97(C8):12731, DOI 10.1029/92JC00912

Jiang L, LeBlond PH (1993) Numerical modeling of an underwater Bingham plastic mudslide and the waves which it generates. Journal of Geophysical Research: Oceans 98(C6):10303-10317

Kelfoun K, Giachetti T, Labazuy P (2010) Landslide-generated tsunamis at Réunion Island. Journal of Geophysical Research: Earth Surface 115(F4)

Lagrée PY, Staron L, Popinet S (2011) The granular column collapse as a continuum: validity of a two-dimensional Navier-Stokes model with a $\mu(\mathrm{I})$-rheology. Journal of Fluid Mechanics 686:378-408

Liu PF, Wu TR, Raichlen F, Synolakis C, Borrero J (2005) Runup and rundown generated by threedimensional sliding masses. Journal of Fluid Mechanics 536:107-144

Lo HY, Liu PLF (2017) On the analytical solutions for water waves generated by a prescribed landslide. Journal of Fluid Mechanics 821:85-116, DOI 10.1017/jfm.2017.251

Lynett P, Liu PLF (2002) A numerical study of submarine-landslide-generated waves and run-up. Proceedings of the Royal Society A: Mathematical, Physical and Engineering Sciences 458(2028):2885-2910, DOI 10.1098/rspa.2002.0973 
Ma G, Kirby JT, Hsu TJ, Shi F (2015) A two-layer granular landslide model for tsunami wave generation: theory and computation. Ocean Modelling 93:40-55

Mangeney-Castelnau A, Vilotte JP, Bristeau MO, Perthame B, Bouchut F, Simeoni C, Yerneni S (2003) Numerical modeling of avalanches based on Saint Venant equations using a kinetic scheme. Journal of Geophysical Research: Solid Earth 108(B11)

Mulligan RP, Take WA (2017) On the transfer of momentum from a granular landslide to a water wave. Coastal Engineering 125:16-22, DOI 10.1016/j.coastaleng.2017.04.001

Pelinovsky E, Poplavsky A (1996) Simplified model of tsunami generation by submarine landslides. Physics and Chemistry of the Earth 21(1-2):13-17

Pouliquen O, Forterre Y (2002) Friction law for dense granular flows: application to the motion of a mass down a rough inclined plane. Journal of Fluid Mechanics 453, DOI 10.1017/S0022112001006796

Ruff LJ (2003) Some aspects of energy balance and tsunami generation by earthquakes and landslides. Pure and Applied Geophysics 160(10-11):2155-2176, DOI 10.1007/s00024-003-2424-y

Sue LP, Nokes RI, Davidson MJ (2011) Tsunami generation by submarine landslides: comparison of physical and numerical models. Environmental Fluid Mechanics 11(2):133-165, DOI 10.1007/ s10652-010-9205-9

Viroulet S (2013) Simulations de tsunamis générés par glissements de terrains aériens. Thèse de doctorat, Aix-Marseille Université, France

Viroulet S, Sauret A, Kimmoun O, Kharif C (2013) Granular collapse into water: toward tsunami landslides. Journal of Visualization 16(3):189-191

Viroulet S, Sauret A, Kimmoun O (2014) Tsunami generated by a granular collapse down a rough inclined plane. EPL (Europhysics Letters) 105(3):34004

Watts P (1997) Water waves generated by underwater landslides. PhD thesis, California Institute of Technology

Watts P (1998) Wavemaker curves for tsunamis generated by underwater landslides. Journal of Waterway, Port, Coastal, and Ocean Engineering 124(3):127-137

Watts P, Grilli ST, Kirby JT, Fryer GJ, Tappin DR (2003) Landslide tsunami case studies using a Boussinesq model and a fully nonlinear tsunami generation model. Natural Hazards and Earth System Science 3(5):391-402, DOI 10.5194/nhess-3-391-2003

Yavari-Ramshe S, Ataie-Ashtiani B (2017) A rigorous finite volume model to simulate subaerial and submarine landslide-generated waves. Landslides 14(1):203-221

Yavari-Ramshe S, Ataie-Ashtiani B (2019) On the effects of landslide deformability and initial submergence on landslide-generated waves. Landslides 16(1):37-53

Zhao T, Utili S, Crosta GB (2016) Rockslide and impulse wave modelling in the Vajont reservoir by DEM-CFD analyses. Rock Mechanics and Rock Engineering 49(6):2437-2456, DOI 10.1007/ s00603-015-0731-0 


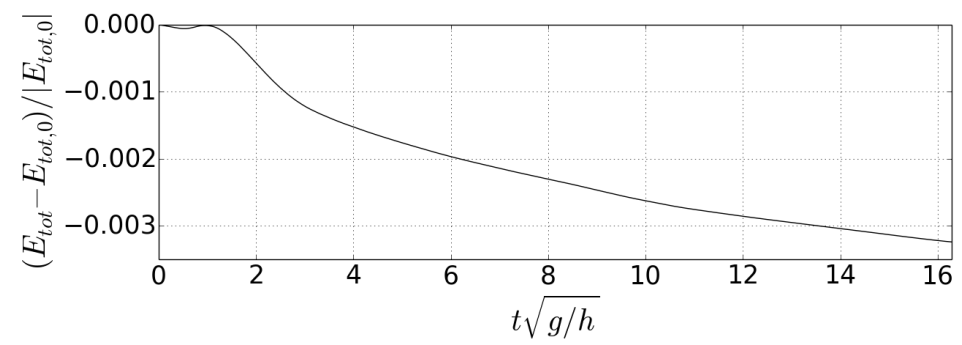

(a)

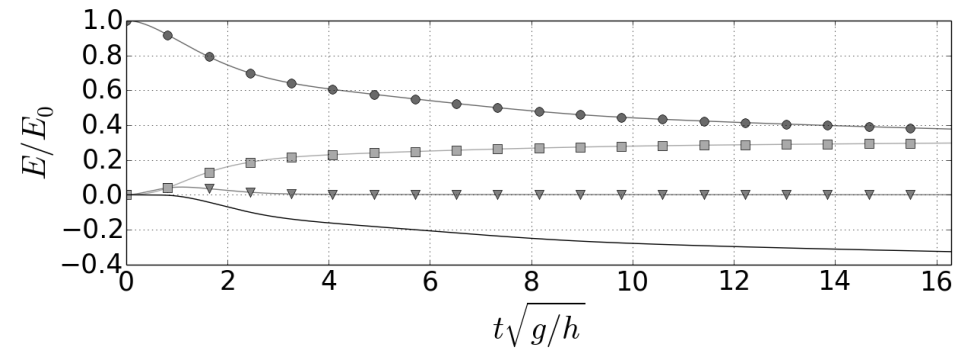

(b)

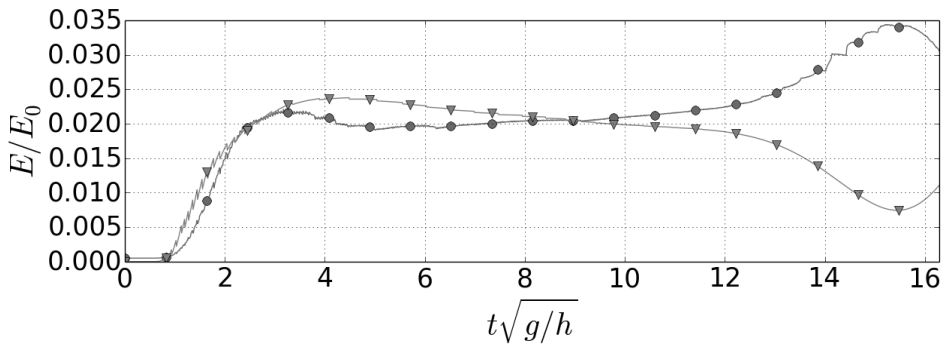

(c)

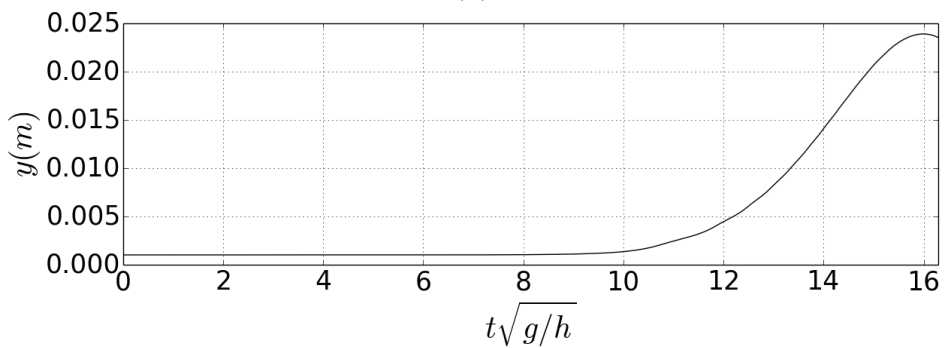

(d)

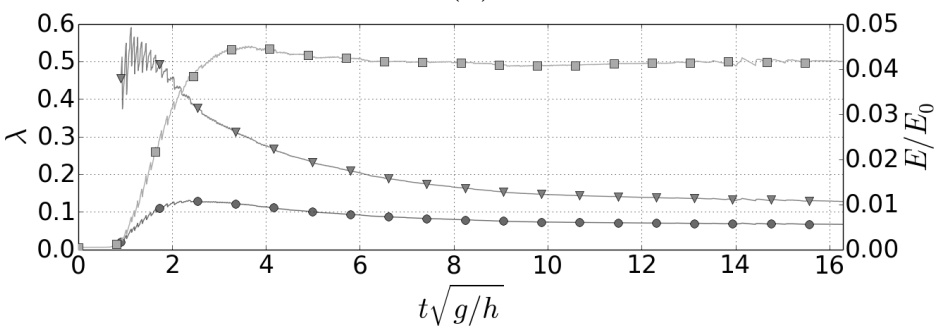

(e)

Figure 9: Time evolution of computed energy components in the subaerial benchmark case (Viroulet et al., 2013) with Newtonian slide $(\eta=10$ Pa.s $)$ ). (a) Relative error on system total energy \{air+water+slide\}, (b) slide potential energy (circles), kinetic energy (triangles) and energy dissipated (squares), energy transferred to water (black), divided by the initial slide potential energy $E_{0}\left(E_{0}=19.7 \mathrm{~J}\right.$ in this case), (c) wave potential energy (circles), kinetic energy (triangles) divided by $E_{0}$, (d) free surface elevation at $x=2 m$, (e) evolution of the wave energy (squares, right axis) divided by $E_{0}$, transfer ratio (left axis) from expression 17 (circles) (Jiang and LeBlond, 1992) and from expression 18 (triangles). 

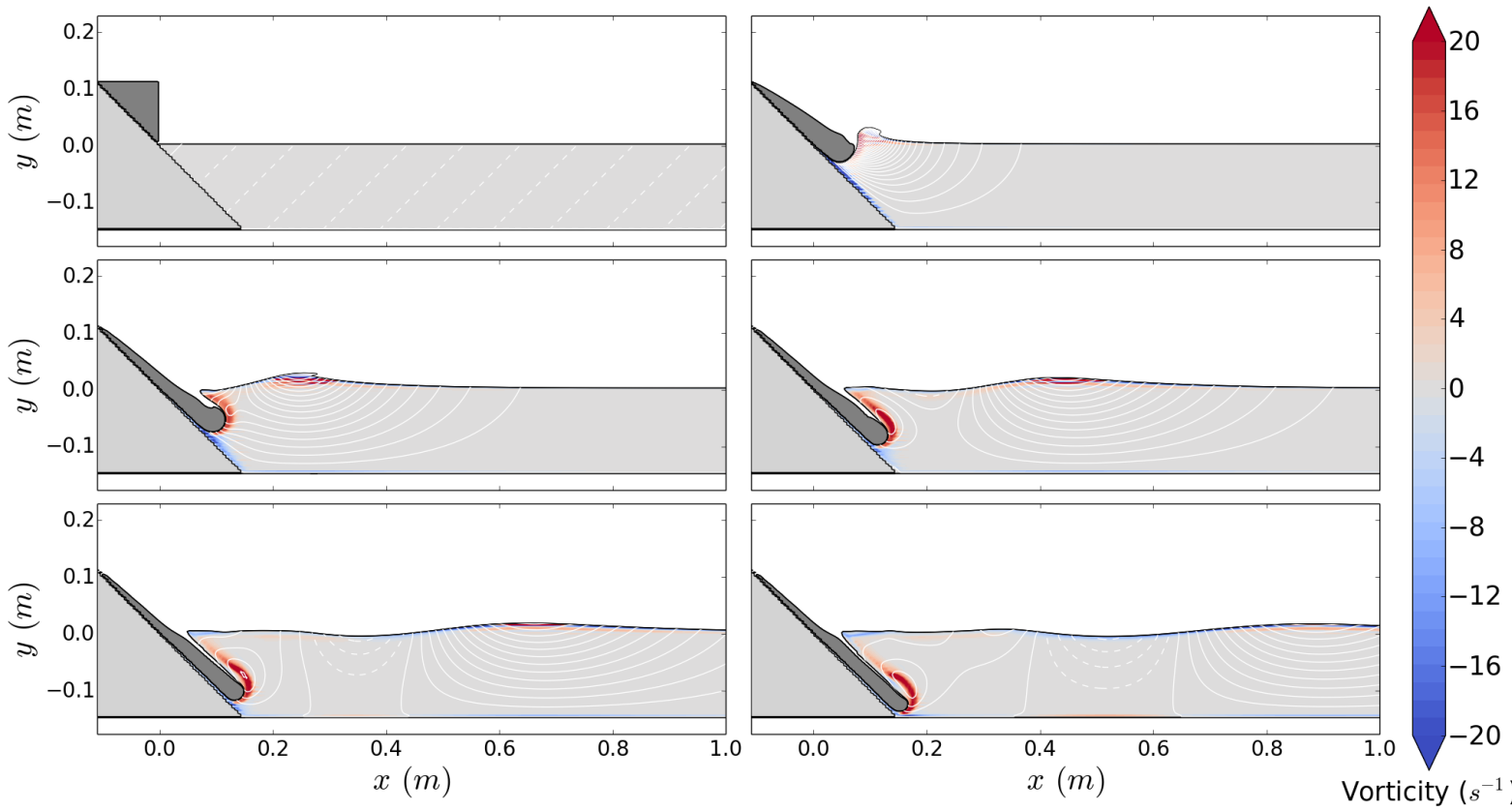

Figure 10: Slide snapshots of the subaerial case with stream contour (white) and water coloured with vorticity, $t=0 s$ in first panel and $0.2 s\left(\Delta t^{*}=1.6\right)$ time difference between figures. 


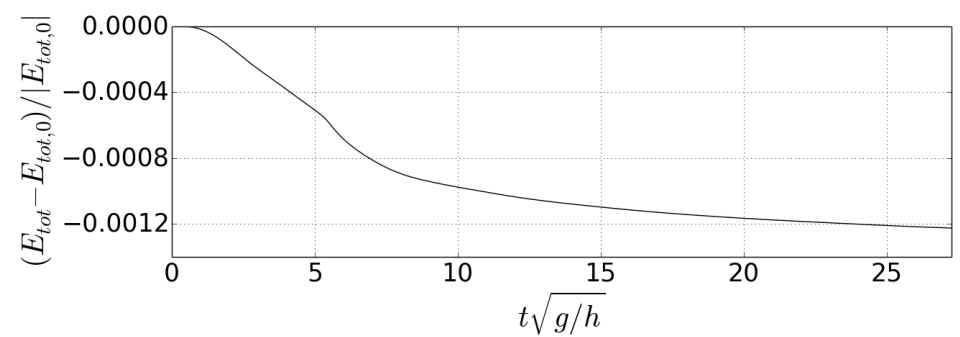

(a)

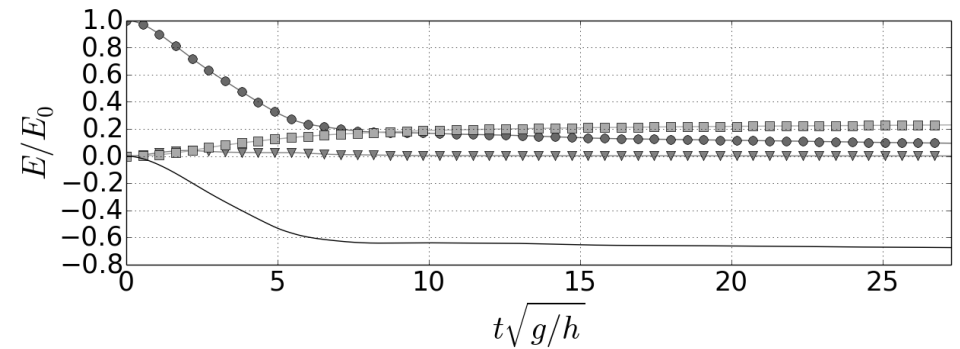

(b)

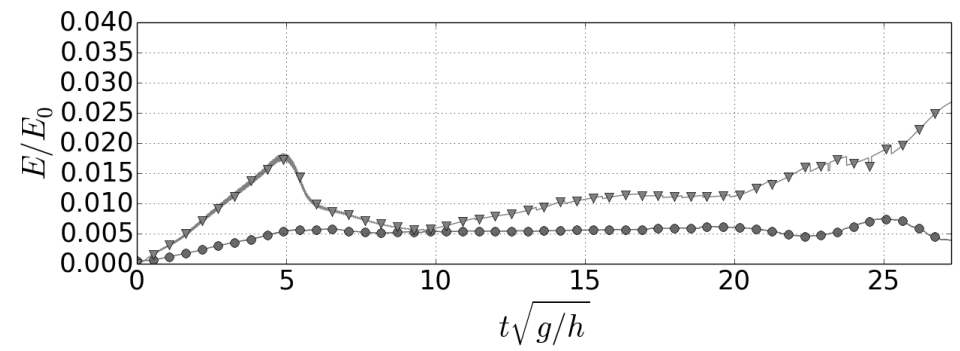

(c)

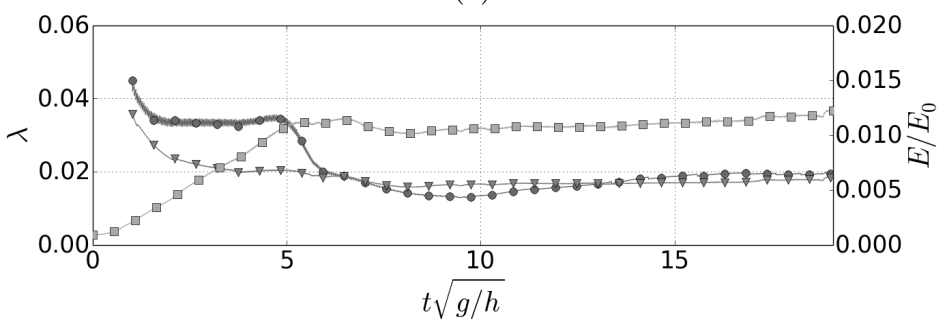

(d)

Figure 11: Time evolution of computed energy components in the submarine benchmark case (Grilli et al., 2017) with Newtonian fluid ( $\eta=1$ Pa.s). (a) Relative error on system total energy \{air+water+slide\}, (b) slide potential energy (circles), kinetic energy (triangles) and energy dissipated (squares), energy transferred to water (black), divided by the initial slide potential energy $E_{0}\left(E_{0}=24.8 \mathrm{~J}\right.$ in this case), (c) wave potential energy (circles), kinetic energy (triangles) divided by $E_{0}$, (d) evolution of the wave energy (squares, right axis) divided by $E_{0}$, transfer ratio (left axis) from expression 17 (circles) (Jiang and LeBlond, 1992) and from expression 18 (triangles). 


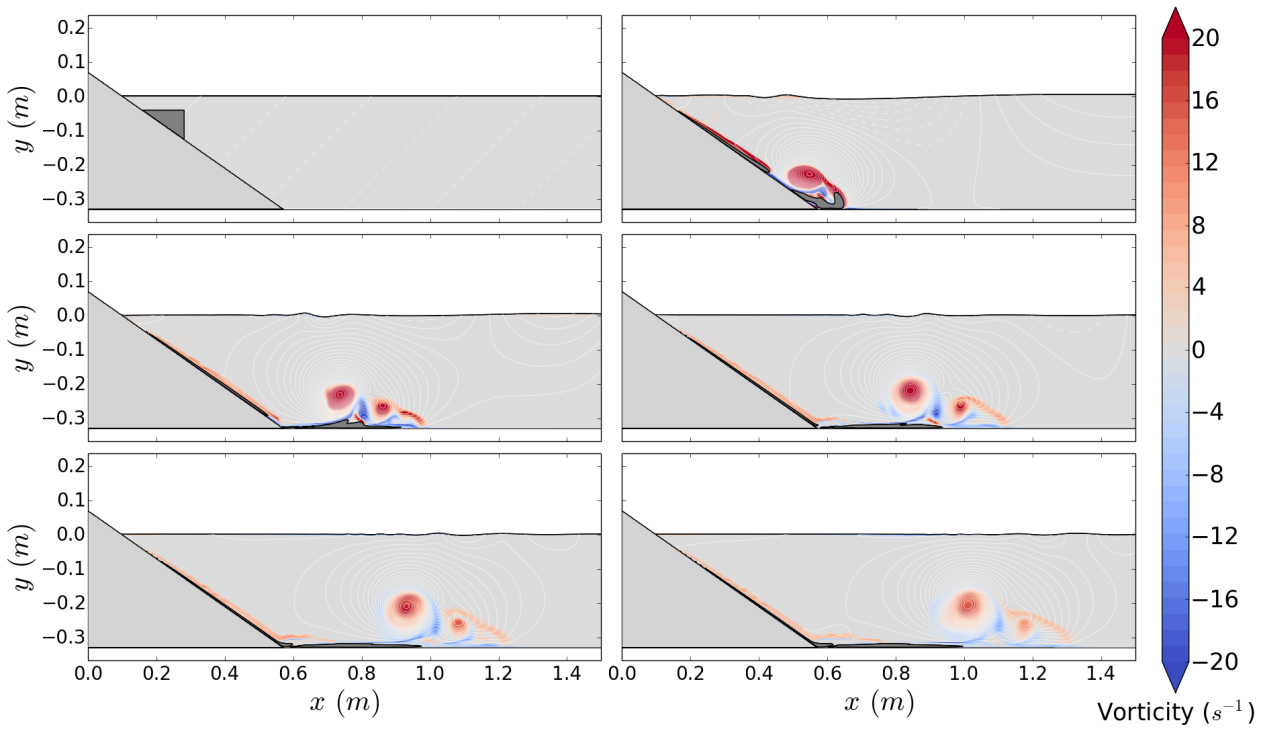

Figure 12: Slide snapshots of the submarine case with stream contour (white) and water coloured with vorticity, $t=0 s$ in first panel and $1 s\left(\Delta t^{*}=5.4\right)$ time difference between figures.

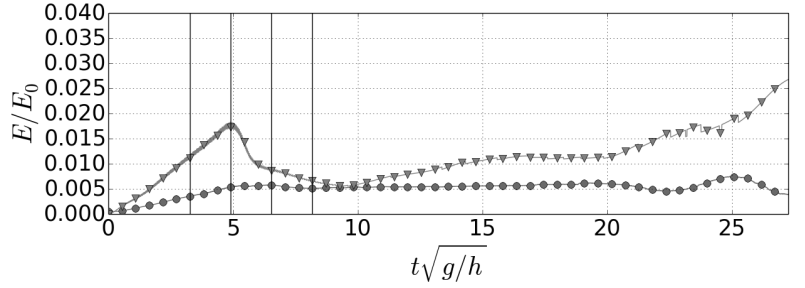

(a)
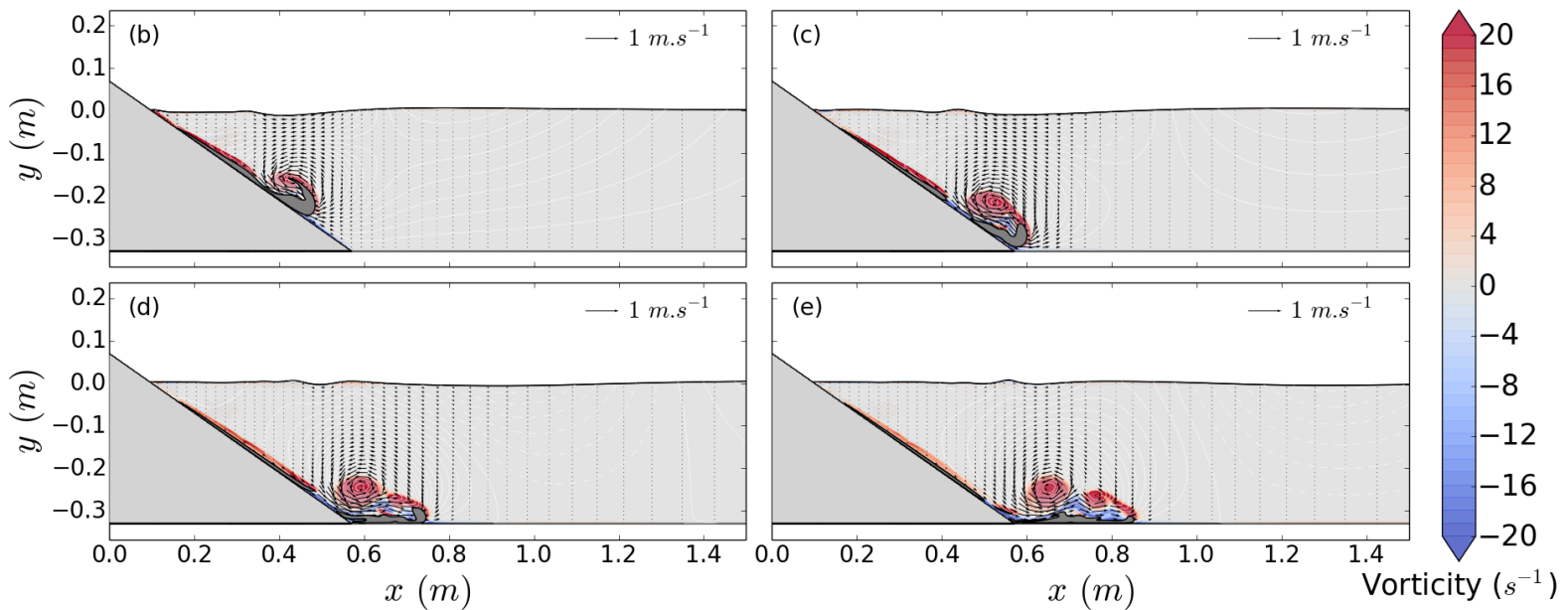

Figure 13: (a) Time evolution of the wave kinetic (triangles) and potential (circles) energy during simulation of the submarine case with the black vertical lines representing the times at which the four following snapshots were taken. (b-e) Slide snapshots with black arrows representing the velocity field at times $t=0.6,0.9,1.2,1.5 s\left(t^{*}=3.3,4.9,6.5,8.2\right)$. 

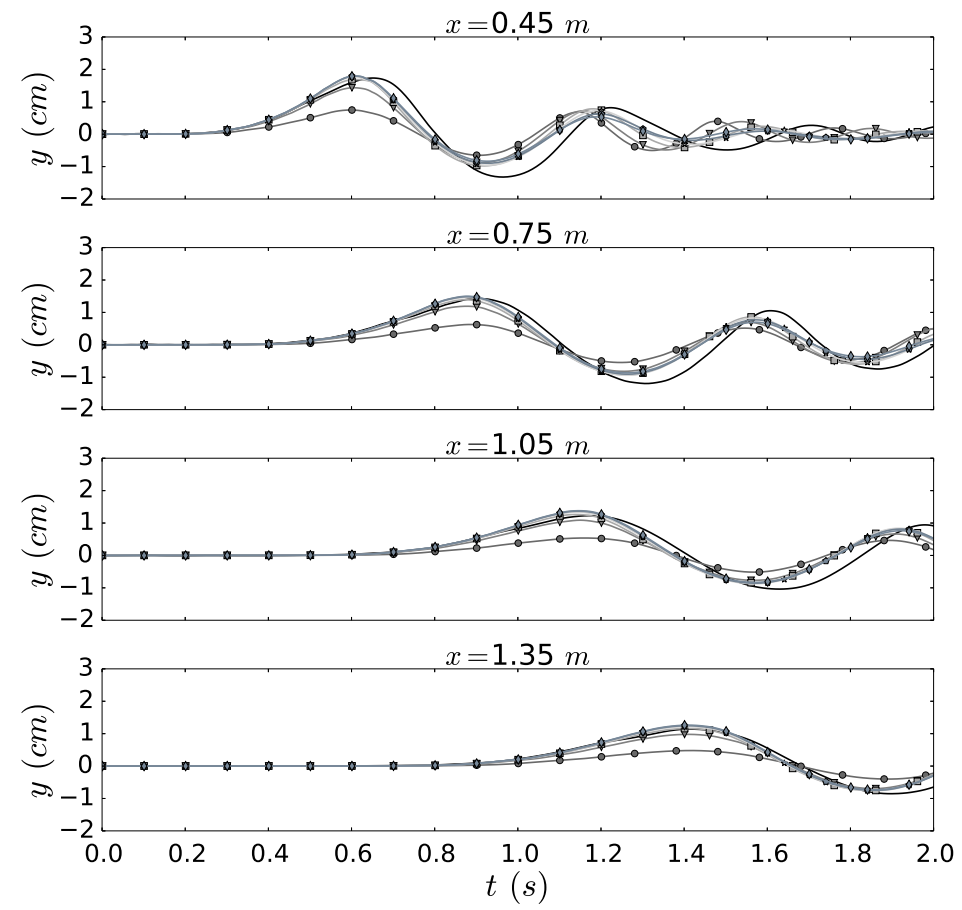

Figure 14: Elevation of the water free surface for the Viroulet's experiment (black line), THETIS simulation with the slide viscosity at $\eta=10$ Pa.s and the slide stopped at $t=0.2 \mathrm{~s}\left(t^{*}=1.6\right)$ (circles), $0.3 \mathrm{~s}$ $\left(t^{*}=2.4\right)$ (triangles), $0.4 \mathrm{~s}\left(t^{*}=3.2\right)$ (squares), $0.5 \mathrm{~s}\left(t^{*}=4\right)$ (stars), $0.6 \mathrm{~s}\left(t^{*}=4.8\right)$ (pentagons), $0.7 \mathrm{~s}$ $\left(t^{*}=5.6\right)$ (diamonds).

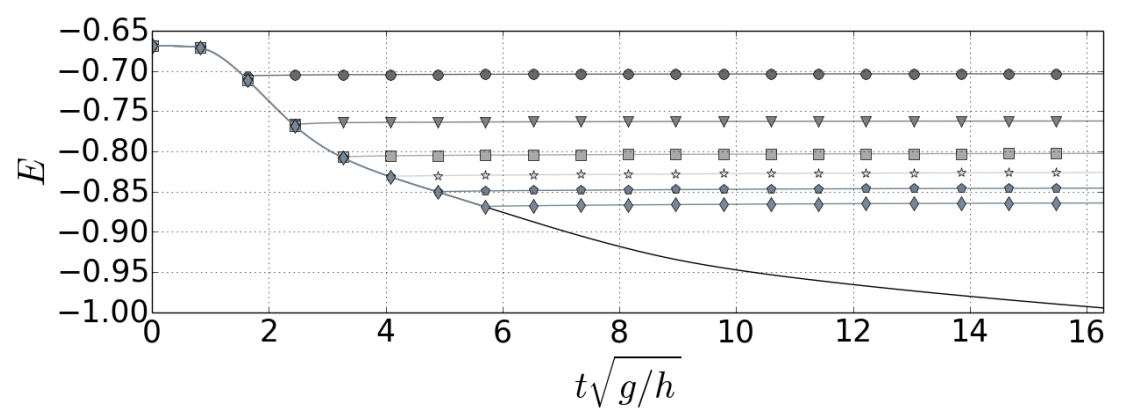

(a)

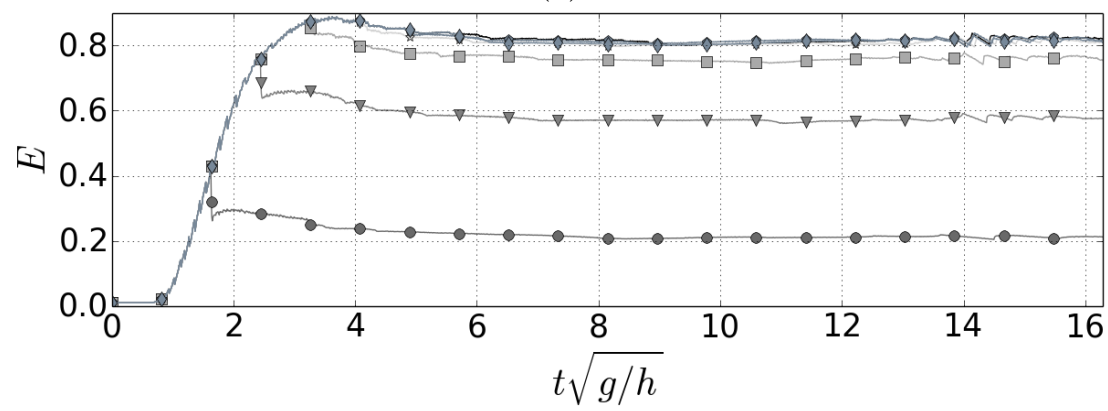

(b)

Figure 15: Time evolution of computed energy transferred by the slide (a) and wave energy (b) with the slide stopped at $t=0.2 \mathrm{~s}\left(t^{*}=1.6\right)$ (circles), $0.3 \mathrm{~s}\left(t^{*}=2.4\right)$ (triangles), $0.4 \mathrm{~s}\left(t^{*}=3.2\right)$ (squares), $0.5 \mathrm{~s}$ $\left(t^{*}=4\right)$ (stars), $0.6 s\left(t^{*}=4.8\right)$ (pentagons), $0.7 s\left(t^{*}=5.6\right)$ (diamonds) and not stopped (black line) in the subaerial case. 

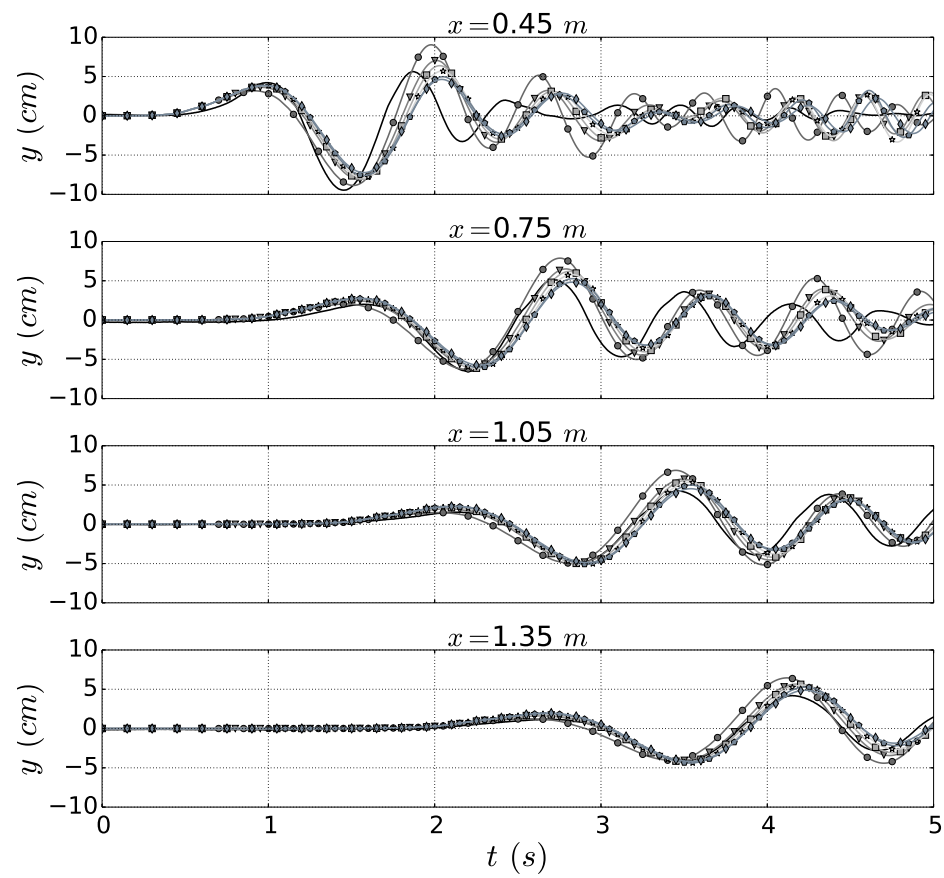

Figure 16: Elevation of the water free surface for the submarine experiment (black line), THETIS simulation with the slide viscosity at $\eta=1$ Pa.s and the slide stopped at $t=0.7 \mathrm{~s}\left(t^{*}=3.8\right)$ (circles), $0.8 \mathrm{~s}$ $\left(t^{*}=4.4\right)$ (triangles), $0.9 \mathrm{~s}\left(t^{*}=4.9\right)$ (squares), $1.0 \mathrm{~s}\left(t^{*}=5.5\right)\left(\right.$ stars), $1.1 \mathrm{~s}\left(t^{*}=6\right)$ (pentagons), $1.2 \mathrm{~s}$ $\left(t^{*}=6.5\right)$ (diamonds). 


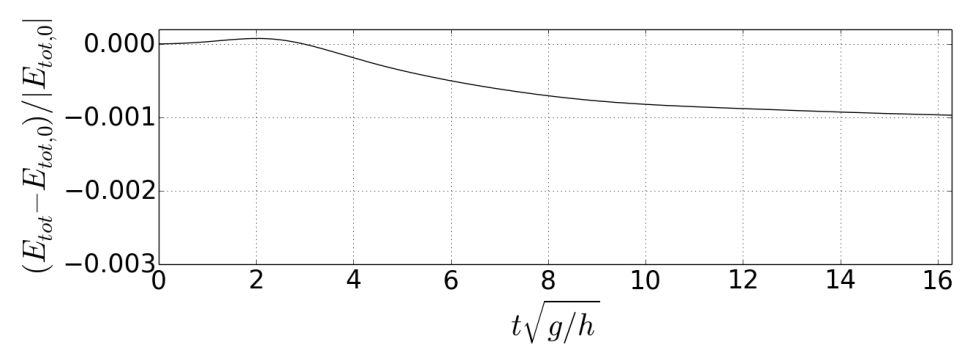

(a)

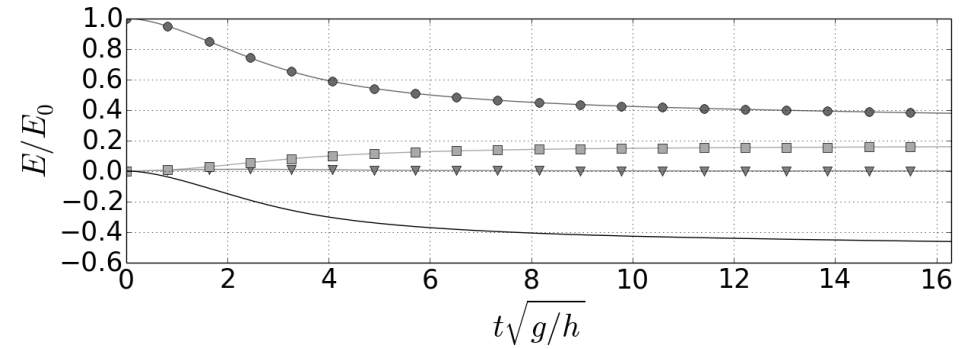

(b)

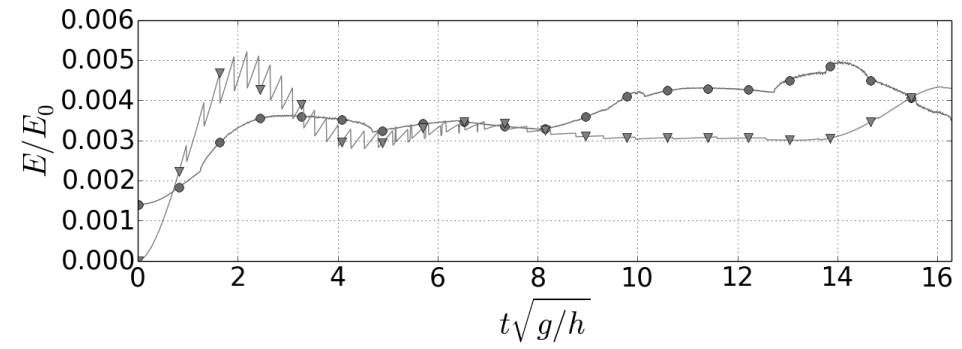

(c)

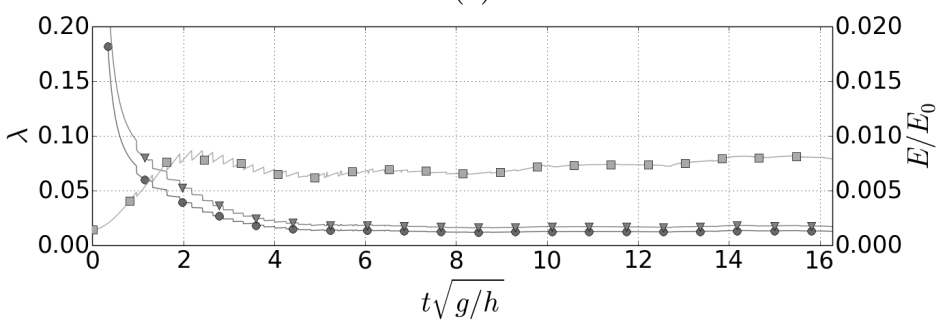

(d)

Figure 17: Time evolution of computed energy components for a simulation case performed with Newtonian slide $(\eta=10$ Pa.s $)$ ) involving the same slide as in Viroulet et al. (2013)'s benchmark case but with the same submergence as in Grilli et al. (2017). (a) Relative error on system total energy \{air+water+slide\}, (b) slide potential energy (circles), kinetic energy (triangles) and energy dissipated (squares), energy transferred to water (black), divided by the initial slide potential energy $E_{0}\left(E_{0}=6.4 J\right.$ in this case), (c) wave potential energy (circles), kinetic energy (triangles) divided by $E_{0}$, (d) evolution of the wave energy (squares, right axis) divided by $E_{0}$, transfer ratio (left axis) from expression 17 (circles) (Jiang and LeBlond, 1992) and from expression 18 (triangles). 